Review

\title{
Current Strategies for Studying the Natural and Synthetic Bioactive Compounds in Food by Chromatographic Separation Techniques
}

\author{
Wioletta Parys *(D), Małgorzata Dołowy (D) and Alina Pyka-Pająk * (D)
}

check for updates

Citation: Parys, W.; Dołowy, M.; Pyka-Pajak, A. Current Strategies for Studying the Natural and Synthetic Bioactive Compounds in Food by Chromatographic Separation Techniques. Processes 2021, 9, 1100. https://doi.org/10.3390/pr9071100

Academic Editor: Francesca Blasi

Received: 23 February 2021

Accepted: 22 June 2021

Published: 24 June 2021

Publisher's Note: MDPI stays neutral with regard to jurisdictional claims in published maps and institutional affiliations.

Copyright: (c) 2021 by the authors. Licensee MDPI, Basel, Switzerland. This article is an open access article distributed under the terms and conditions of the Creative Commons Attribution (CC BY) license (https:// creativecommons.org/licenses/by/ $4.0 /)$.
Department of Analytical Chemistry, Faculty of Pharmaceutical Sciences in Sosnowiec, Medical University of Silesia in Katowice, Jagiellońska 4, 41-200 Sosnowiec, Poland; mdolowy@sum.edu.pl

* Correspondence: wparys@sum.edu.pl (W.P.); apyka@sum.edu.pl (A.P.-P.); Tel.: +48-32-364-15-34 (W.P.); +48-32-364-15-30 (A.P.-P.)

\begin{abstract}
The present study summarizes the new strategies including advanced equipment and validation parameters of liquid and gas chromatography methods i.e., thin-layer chromatography (TLC), column liquid chromatography (CLC), and gas chromatography (GC) suitable for the identification and quantitative determination of different natural and synthetic bioactive compounds present in food and food products, which play an important role in human health, within the period of 2019-2021 (January). Full characteristic of some of these procedures with their validation parameters is discussed in this work. The present review confirms the vital role of HPLC methodology in combination with different detection modes i.e., HPLC-UV, HPLC-DAD, HPLC-MS, and HPLC-MS/MS for the determination of natural and synthetic bioactive molecules for different purposes i.e., to characterize the chemical composition of food as well as in the multi-residue analysis of pesticides, NSAIDs, antibiotics, steroids, and others in food and food products.
\end{abstract}

Keywords: bioactive compounds; food; separation techniques; liquid chromatography; gas chromatography

\section{Introduction}

Bioactive compounds are natural or synthetic (partially or totally) compounds that show biological activity i.e., have the ability to interact with living tissues and indicate an effect on human body including the promotion of good health, thus they are important as new ingredients of the current functional food (e.g., antioxidants) [1]. Food samples are very complex mixtures consisting not only of naturally occurring bioactive compounds with beneficial role on human health like for example vitamins, minerals, antioxidants but other substances coming from agrochemical treatments i.e., pesticides as well as promotors animals growth or veterinary drugs. Therefore monitoring the level of different veterinary drugs or organic pesticides coming from agrochemical treatments in food and food products could ensure the safety of potential consumers. Natural and synthetic bioactive compounds occur in foods in small quantities and represent a wide group of chemical compounds. Because of the complexity of food matrices, the separation and next accurate determination of their bioactive constituents with different chemical structure requires an universal analytical methodology like liquid and gas chromatography or combination of both chromatographic techniques.

For this fact, this article reviews new strategies including advanced equipment and validation parameters of liquid and gas chromatography methods dedicated for the identification and quantitative analysis of natural and synthetic bioactive compounds occurring in food and food products within the period of 2019-2021 (January). Special attention is given to optimization including the validation process of chromatographic analysis performed by using thin-layer chromatography (TLC), high-performance liquid chromatography 
(HPLC), and gas chromatography (GC) coupled with different detection modes ((TLCUV/Vis, TLC-densitometry, HPTLC-MS, HPLC-UV/Vis, HPLC-DAD(PDA), HPLC-MS, HPLC-MS/MS, HPLC-TQ-ESI-MS/MS, GC-MS, GC-MS/MS, GC-CPI-MS/MS)) as well as the combined chromatographic techniques e.g., HPLC/GC that may be valuable for the separation, screening, quantitative determination or evaluation of certain physicochemical and pharmacological properties of many including the newly developed natural and synthetic bioactive compounds in food and food products.

\section{Thin Layer Chromatography}

Liquid chromatography, including thin-layer chromatography, along with other chromatographic techniques, is one of the most popular methods used in the current analysis of bioorganic and bioinorganic compounds in different including food samples [2-15].

\section{TLC Analysis of Selected Bioactive Compounds in Food Samples}

The recently published papers indicate that thin-layer chromatography was successfully used for the quantification of selected antibiotics, alkaloids, aromatic amines, and gallic acid in food [2-5]. Both i.e., contact and immersion TLC-bioautography with the use of silica gel $\mathrm{F}_{254}$ plates, $7.5 \%$ of $\mathrm{KH}_{2} \mathrm{PO}_{4}$, and Escherichia coli ATCC 8739 as a test bacterium were employed for the sensitive determination of streptomycin in the presence of kanamycin sulfate in frozen shrimp, thus to control the antibiotic abuse in frozen food [2]. The work of Foudah et al. [3] shows a rapid and sensitive HPTLC method with densitometry for the quantification of trigonelline content as important bioactive constituent of Arabic coffees at the level of ng/spot [3]. Another study [4] indicates the use of HPTLC-DPPH (high-performance thin-layer chromatography coupled with the use of 2,2-diphenyl-1-picrylhydrazyl) method for rapid and simple screening of antioxidant constituents i.e., gallic acid in honey, in natural food products. Similarly, the study of Piszcz and coworkers [6] demonstrated the ability of TLC method to separate two different forms of DPPH (i.e., DPPH and DPPH-H) and also for the measurement of total antioxidant potential in the meat samples. Another study describes a novel and fully validated HPTLC-MS method for the rapid identification and determination of toxic aryl azo amines in food matrices. The achieved level of detection and quantification of these compounds was in ppm [5].

Another authors; Turkmen and Kurada [7] confirmed the utility of HPTLC on silica gel $60 \mathrm{~F}_{254}$ plates with densitometric measurements to asses next toxic compound, namely patulin as contamination of fruit-based baby foods in Turkey.

In the vast majority of analyzes, fatty acids are investigated using the GC technique as fatty acid methyl esters. However, Dabrowska et al. [8] developed a TLC method in combination with densitometry for the determination of omega-3 fatty acids: linolenic (ALA), docosahexaenoic (DHA), and eicosapentaenoic acids (EPA) in 15 dietary supplements and 5 cooking products.

Some studies indicate the important role of TLC and HPTLC methods as comprehensive techniques for the detection and identification of pesticides and the toxicity caused by these compounds [9-15]. Several new chromogenic reagents have been reported in the literature such as diphenylamine reagent for detection of organochloro insecticide endosulfan [9], stannous chloride and hydrochloric acid (reducing reagent) followed by a sodium nitrite in hydrochloric acid (coupling reagent) and $\beta$-napthol in sodium hydroxide for the detection of herbicide oxyfluorten [10], chloranil reagent with nitric acid for detection of organophosporus insecticide monocrotophos [11], 4-amminoantipyrene reagent with potassium ferricyanide for detection and identification of 2,4-dichlorophenol, an intermediate of 2,4-D (2,4-dichlorophenoxyacetic acid) herbicide [12], cupric acetate reagent for detection of organophosphate insecticide profenofos [13], and cobalt thiocyanate reagent for detection of organophosporus herbicide glyphosate [14]. Hussain et al. [15] developed an HPTLC method for the determination of residues of various pesticides in brinjal samples 
from a market of Pakistan. The authors showed that HPTLC can be an alternative method to HPLC for the detection of pesticide residues.

The scientific literature cited and discussed above indicates that TLC/HPTLC can be successfully used to detect and quantify a wide variety of synthetic and natural classes of bioactive compounds occurring in food and food products. There are many reports in the scientific literature combining TLC with a densitometry. However, there is an increase of works linking TLC with MS. Therefore, it seems that in the next few years there should be more scientific papers using TLC/MS.

\section{Column Liquid Chromatography}

Extensive review of literature published in the two last years indicates that highperformance liquid chromatography (HPLC) with different detection systems such as ultraviolet detector (HPLC-UV), photodiode array detector (HPLC-PDA), or coupled to mass spectrometry or tandem mass spectrometry called as HPLC-MS and HPLCMS/MS respectively is a powerful analytical tool with many applications including food analysis [16-66].

\section{Column Liqiud Chromatography in Analysis of Selected Bioactive Compounds in Food Samples}

Due the widespread use of agricultural chemicals in food production, people are exposed to low levels of pesticide residues through their diets. Because the organic pesticides usually exist in very small amounts in food samples and have different chemical structure containing, for example, triazine, imidazolinone, phenyluracyl, or macrocyclic lactone structure, thus there is a need to develop efficient and sensitive CLC systems for the simultaneous determination of compounds that are dangerous to human health, present in food and food products which belong to one of the presented groups as well as to various groups (i.e., multiclass pesticides) [31,32]. Table 1 shows the utility of selected CLC procedures with validation parameters that have been applied in analysis of food samples [16-48]. The current literature review indicates that validated high performance liquid chromatography is a powerful analytical technique used to determine many single or multi-class pesticides present in different food matrices. Most developed methods were validated according to the European SANTE guidelines (SANTE/11945/2015, SANTE/11813/2017, SANTE/12682/2019) in terms of linearity, LOD, LOQ, accuracy, recovery, and precision, as shown in Table 1. As it can be observed, liquid chromatography is particularly appropriate for the analysis of polar, non-volatile, and/or thermally labile pesticides. Because of its high selectivity and sensitivity, HPLC and UHPLC in combination with MS/MS have mostly been used in this field especially to determine the insecticides and herbicides belonging to organophosphorus compounds, imidazolinone and pyridine carboxylic acid derivatives, and in study of samples containing multiclass pesticides [16-24,27,30-34]. However, in a few cases, i.e., triazine and phenylurea herbicides, the HPLC coupled with spectrophotometric detection HPLC-UV or DAD has also been applied $[25,26,28]$. Various kinds of stationary phases (columns) have been used in the HPLC determination of pesticides, mainly C18 [16-18,20,25-31], and also chiral [19,33], BEH HILIC [21], Hypercarb [22], Obelisc N HILIC [24,34], Acquity UPLC HSS T3 [23,32]. In general, water or water with formic acid or acetic acid or ammonium formate, acetonitrile, and methanol have been applied as mobile phases with gradient or isocratic elution, respectively. 
Table 1. Column liquid chromatography in food analysis.

\begin{tabular}{|c|c|c|c|}
\hline Matrix/Compound & Chromatographic Conditions & Other Parameters & Refs. \\
\hline \multicolumn{4}{|c|}{ Variety Classes of Pesticides } \\
\hline \multicolumn{4}{|c|}{ Insecticides } \\
\hline \multicolumn{4}{|c|}{ Containing macrocyclic lactone structure } \\
\hline $\begin{array}{l}\text { Porcine muscle, egg, milk, } \\
\text { eel, flatfish, shrimp } \\
\text { Spinosyn A (SPA), } \\
\text { Spinosyn D (SPD), } \\
\text { Temephos (TP), } \\
\text { Piperonyl butoxide (PB) }\end{array}$ & $\begin{array}{c}\text { LC-TQ-ESI-MS/MS } \\
\text { Multiple reaction monitoring (MRM) mode } \\
\text { Phenomenex Kinetex EVO C18 } \\
(150 \times 2.1 \mathrm{~mm}, 2.6 \mu \mathrm{m}) \\
\text { Eluent A: } 0.1 \% \text { formic acid in } 10 \mathrm{mM} \\
\text { ammonium formate in distilled water; } \\
\text { Eluent B: methanol } \\
\text { A:B }(10: 90, v / v) \\
\text { Flow rate: } 0.2 \mathrm{~mL} / \mathrm{min}\end{array}$ & $\begin{array}{c}\text { Linearity }(\mu \mathrm{g} / \mathrm{kg}): \\
3.5 \div 35 \text { (for SPA), } 1.5 \div 15 \text { (for SPD) } \\
5 \div 50 \text { (for TP, PB) } \\
\text { LOD }(\mu \mathrm{g} / \mathrm{kg}): \\
0.5 \div 0.8 \text { (for SPA), } 0.1 \text { (for SPD) } \\
1.1 \div 1.6 \text { (for TP), } 0.3 \div 0.7 \text { (for PB) } \\
\text { Recovery: } 70 \div 105 \%\end{array}$ & [16] \\
\hline \multicolumn{4}{|c|}{ Organothiophosphate derivatives } \\
\hline $\begin{array}{c}\text { Tomato, cabbage, barley, } \\
\text { Xijiang river water, tap water } \\
\text { Quinalphos(QP), } \\
\text { Triazophos (TZ), } \\
\text { Parathion (PTN), } \\
\text { Fenthion (FT), } \\
\text { Chlorpyrifos-methyl (CHM) }\end{array}$ & $\begin{array}{c}\text { HPLC-UV } \\
\lambda=254 \mathrm{~nm} \\
\text { Agilent TC-C18 } \\
(150 \times 4.6 \mathrm{~mm}, 5 \mu \mathrm{m}) \\
\text { Pure methanol } \\
\text { Flow rate: } 0.5 \mathrm{~mL} / \mathrm{min}\end{array}$ & $\begin{array}{c}\text { Linearity: } \\
0.02 \div 2.00 \mu \mathrm{g} / \mathrm{mL} \\
\text { LOD }(\mu \mathrm{g} / \mathrm{L}): \\
3.0 \text { (for QP), } 5.0 \text { (for TZ, PTN) } \\
6.0 \text { (for FT), } 10.0 \text { (for CHM) } \\
\text { Recovery: } 80 \div 98 \%\end{array}$ & [17] \\
\hline
\end{tabular}

\section{Herbicides}

Phenoxyacetic acid derivatives

\section{HPLC-TQ-ESI-MS/MS}

Multiple reaction monitoring (MRM) mode RP C18

Corn, wheat, rice

Phenoxy acid herbicides (6)
$(150 \times 2.1 \mathrm{~mm}, 3.5 \mu \mathrm{m})$ Eluent A: water;

Eluent B: acetonitrile

Gradient elution

Flow rate: $0.3 \mathrm{~mL} / \mathrm{min}$
Linearity: $0.200 \div 40.0 \mu \mathrm{g} / \mathrm{kg}$

LOD: $0.0500 \div 0.300 \mu \mathrm{g} / \mathrm{kg}$

Accuracy: $95.6 \div 107 \%$

Intraday precision: $0.895 \div 5.40 \%$

Interday precision: $1.13 \div 6.61 \%$

Recovery: $73.8 \div 115 \%$

Imidazolinone derivatives

Soybean, peanut, wheat, maize, rice

$S$-imazethapyr (SIT)

$R$-imazethapyr (RIT)

$S$-imazamox (SIZ)

$R$-imazamox (RIZ)

$S$-imazapic (SIP)

$R$-imazapic (RIP)
UPLC-TQ-ESI-MS/MS

Multiple reaction monitoring (MRM) mode

Chiralcel OJ-3R

$(150 \times 4.6 \mathrm{~mm}, 3 \mu \mathrm{m})$

Eluent A: $0.1 \%$ formic acid aqueous solution;

Eluent B: acetonitrile

Gradient elution

Flow rate: $0.4 \mathrm{~mL} / \mathrm{min}$
$\mathrm{LOD}(\mu \mathrm{g} / \mathrm{kg})$ :

$0.35 \div 0.48$ (for SIP), $0.36 \div 0.72$ (for RIP)

$0.40 \div 0.88$ (for SIT), $0.34 \div 0.75$ (for RIT)

$1.0 \div 1.5$ (for SIZ), $0.98 \div 1.4$ (for RIZ)

Recovery: $64.2 \div 106.4 \%$

Pyridine carboxylic acid derivatives

LC-TQ-ESI-MS/MS

Multiple reaction monitoring (MRM) mode

Milk Waters Xselect HSS T3 (C18)

$(2.1 \times 150 \mathrm{~mm}, 5 \mu \mathrm{m})$

aminopyralid, picloram,

Eluent A: ultrapure water;

Eluent B: methanol

Linearity: $1 \div 50 \mu \mathrm{g} / \mathrm{L}$

LOD: $0.124 \mu \mathrm{g} / \mathrm{L}$

fluroxypyr, clopyralid

Gradient elution

Recovery: $75.3 \div 89.8 \%$

Flow rate: $300 \mu \mathrm{L} / \mathrm{min}$ 
Table 1. Cont.

\begin{tabular}{|c|c|c|c|}
\hline Matrix/Compound & Chromatographic Conditions & Other Parameters & Refs. \\
\hline \multicolumn{4}{|c|}{ Quaternary ammonium salt derivatives } \\
\hline $\begin{array}{c}\text { Barley, wheat } \\
\text { Paraquat (PQ), } \\
\text { Diquat (DQ), Chlormequat } \\
\text { CHQ), Mepiquat (MQ) }\end{array}$ & $\begin{array}{c}\text { UHPLC-TQ-ESI-MS/MS } \\
\text { Selected reaction monitoring (SRM) mode } \\
\text { Acquity UPLC }{ }^{\mathrm{TM}} \text { BEH HILIC } \\
(100 \times 2.1 \mathrm{~mm}, 1.7 \mu \mathrm{m}) \\
\text { Eluent A: aqueous solution of ammonium } \\
\text { formate } 60 \mathrm{mmol} / \mathrm{L} \text { at } \mathrm{pH} 3.7 \\
\text { Eluent } \mathrm{B}: \text { acetonitrile } \\
\text { A:B }(40: 60, v / v) \\
\text { Flow rate: } 0.250 \mathrm{~mL} / \mathrm{min}\end{array}$ & $\begin{array}{c}\text { Linearity }(\mu \mathrm{g} / \mathrm{kg}): \\
80 \div 1000 \text { (for } \mathrm{CHQ}), 40 \div 1000 \text { (for MQ) } \\
20 \div 1000 \text { (for } P Q, \mathrm{DQ}) \\
\text { LOD }(\mu \mathrm{g} / \mathrm{kg}): \\
24 \text { (for } \mathrm{CHQ}), 12 \text { (for MQ), } \\
6 \text { (for PQ, DQ) } \\
\text { Recovery: } 93 \div 106 \%\end{array}$ & {$[21]$} \\
\hline \multicolumn{4}{|c|}{ Organophosphorus compounds, chlorates } \\
\hline $\begin{array}{c}\text { Fruits, vegetables, } \\
\text { infant foods } \\
\text { Glyphosate (GLY), } \\
\text { Aminomethyl phosphonic } \\
\text { acid (AMPA), } \\
\text { Phosphonic acid (PHA), } \\
\text { Fosetyl-Al (FAL), } \\
\text { Chlorate (CHL), } \\
\text { Perchlorate (PCH) }\end{array}$ & $\begin{array}{c}\text { UHPLC-Q Orbitrap-ESI-MS/MS } \\
\text { Thermo Scientific Hypercarb } \\
(3 \times 100 \mathrm{~mm}, 5 \mu \mathrm{m}) \\
\text { Eluent A: } 0.4 \% \text { formic acid in methanol; } \\
\text { Eluent B: } 0.4 \% \text { formic acid in purified water } \\
\text { A:B }(95: 5, v / v) \\
\text { Flow rate: } 0.3 \mathrm{~mL} / \mathrm{min}\end{array}$ & $\begin{array}{c}\text { Linearity: } 0.001 \div 0.1 \mathrm{mg} / \mathrm{L} \\
\text { LOQ }(\mathrm{mg} / \mathrm{kg}): \\
0.0004 \text { (for PCH) } \\
0.001 \text { (for FAL) } \\
0.002 \text { (for CHL) } \\
0.003 \text { (for GLY, AMPA) } \\
0.004 \text { (for PHA) } \\
\text { Recovery: } 72 \div 116 \%\end{array}$ & [22] \\
\hline $\begin{array}{c}\text { Corn } \\
\text { Glyphosate, Glufosinate }\end{array}$ & $\begin{array}{c}\text { UHPLC-ESI-QTRAP-MS } \\
\text { Multiple reaction monitoring (MRM) mode } \\
\text { Acquity UPLC HSS T3 } \\
(2 \times 100 \mathrm{~mm}, 1.8 \mu \mathrm{m}) \\
\text { Eluent A: } 0.05 \% \text { ammonia water; } \\
\text { Eluent B: acetonitrile } \\
\text { A:B }(90: 10, v / v) \\
\text { Flow rate: } 0.2 \mathrm{~mL} / \mathrm{min}\end{array}$ & $\begin{array}{c}\text { Linearity: } 10.0 \div 500 \mathrm{ng} / \mathrm{mL} \\
\text { LOD: } 0.0015 \mathrm{mg} / \mathrm{kg} \\
\text { Recovery: } 90.3 \div 95.4 \% \\
\text { Intraday precision: } 1.24 \div 3.35 \% \\
\text { Interday precision: } 3.56 \div 6.06 \%\end{array}$ & [23] \\
\hline $\begin{array}{l}\text { Vegetable milk, } \\
\text { beer, wine } \\
\text { Highly polar pesticides } \\
\text { (14) including: } \\
\text { glyphosate, glufosinate, } \\
\text { ethephon, fosetyl } \\
\text { and metabolites }\end{array}$ & $\begin{array}{c}\text { LC-ESI-QTRAP-MS } \\
\text { Multiple reaction monitoring (MRM) mode } \\
\text { Obelisc N HILIC } \\
(150 \times 2.1 \mathrm{~mm}, 5 \mu \mathrm{m}) \\
\text { Eluent A: water with } 1 \% \text { formic acid; } \\
\text { Eluent B: acetonitrile } \\
\text { Gradient elution } \\
\text { Flow rate: } 0.5 \mathrm{~mL} / \mathrm{min}\end{array}$ & $\begin{array}{c}\text { Linearity: } 0.2 \div 50 \mathrm{ng} / \mathrm{mL} \\
\text { ILOD (instrumental LOD): } 0.2 \mathrm{ng} / \mathrm{mL} \\
\text { Recovery: } 70 \div 120 \%\end{array}$ & [24] \\
\hline \multicolumn{4}{|c|}{ Triazine compounds/chlorinated anilide derivatives } \\
\hline $\begin{array}{l}\text { White gourd, tomato, } \\
\text { soybean milk } \\
\text { Metribuzin, Simetryn, } \\
\text { Propazine, Prometryne }\end{array}$ & $\begin{array}{c}\text { HPLC-DAD } \\
\lambda=222 \mathrm{~nm} \\
\text { Centurysil C18 } \\
(200 \times 4.6 \mathrm{~mm}, 5 \mu \mathrm{m}) \\
\text { Eluent A: acetonitrile; } \\
\text { Eluent B: water } \\
\text { A:B }(55: 45, v / v) \\
\text { Flow rate: } 1.0 \mathrm{~mL} / \mathrm{min}\end{array}$ & $\begin{array}{l}\text { Linearity: } 0.3 \div 100.0 \mathrm{ng} / \mathrm{g} \text { for white } \\
\text { gourd and tomato } \\
\text { Linearity: } 0.5 \div 100 \mathrm{ng} / \mathrm{mL} \text { for } \\
\text { soybean milk } \\
\text { LOD: } 0.10 \div 0.20 \mathrm{ng} / \mathrm{g} \text { for white gourd } \\
\text { and tomato } \\
\text { LOD: } 0.15 \div 0.30 \mathrm{ng} / \mathrm{mL} \text { for } \\
\text { soybean milk }\end{array}$ & [25] \\
\hline $\begin{array}{c}\text { Beans } \\
\text { Atrazine (AZ), } \\
\text { Oxadiazon (OZ), } \\
\text { Metazachlor (MZ), } \\
\text { Propanil (P) }\end{array}$ & $\begin{array}{c}\text { HPLC-DAD } \\
\lambda=230 \mathrm{~nm} \\
\text { Aqilent Eclipse XDB-C18 } \\
(150 \times 4.6 \mathrm{~mm}, 3.5 \mu \mathrm{m}) \\
\text { Eluent A: water; } \\
\text { Eluent B: acetonitrile } \\
\text { Gradient elution } \\
\text { Flow rate: } 0.50 \mathrm{~mL} / \mathrm{min}\end{array}$ & $\begin{array}{c}\text { Linearity: } 0.1 \div 10 \mu \mathrm{g} / \mathrm{mL} \\
\text { LOD }(\mu \mathrm{g} / \mathrm{kg}): \\
10.3(\text { for } \mathrm{AZ}) \\
2.4(\text { for OZ) } \\
2.9 \text { (for MZ) } \\
3.8 \text { (for P) } \\
\text { Recovery: } 90.7 \div 116.5 \%\end{array}$ & [26] \\
\hline
\end{tabular}


Table 1. Cont.

\begin{tabular}{|c|c|c|c|}
\hline Matrix/Compound & Chromatographic Conditions & Other Parameters & Refs. \\
\hline \multicolumn{4}{|c|}{ Acidic herbicides } \\
\hline $\begin{array}{l}\text { Cucumber, orange } \\
\text { Acidic herbicides (27) } \\
\text { Phytohormones (8) }\end{array}$ & $\begin{array}{c}\text { UHPLC-TQ-ESI-MS/MS } \\
\text { Multiple reaction monitoring (MRM) mode } \\
\text { Acquity UPLC BEH C-18 } \\
(100 \times 2.1 \mathrm{~mm}, 1.7 \mu \mathrm{m}) \\
\text { Eluent A: } 1 \% \text { acetic acid and } 5 \% \\
\text { methanol in water; } \\
\text { Eluent B: } 1 \% \text { acetic acid in methanol } \\
\text { Gradient elution } \\
\text { Flow rate: } 0.35 \mathrm{~mL} / \mathrm{min}\end{array}$ & $\begin{array}{c}\text { For all compounds: } \\
\text { Linearity: } 10 \div 150 \mu \mathrm{g} / \mathrm{kg} \\
\text { LOQ: } 10 \mu \mathrm{g} / \mathrm{kg} \\
\text { Recovery: } 86 \div 120 \% \\
\text { Intraday precision: } 1 \div 20 \% \\
\text { Interday precision: } 4 \div 20 \%\end{array}$ & [27] \\
\hline \multicolumn{4}{|c|}{ Phenylurea derivatives } \\
\hline $\begin{array}{c}\text { Soybean milk, tomato } \\
\text { Metoxuron, Monuron, } \\
\text { Chlortoluron, Monolinuron, } \\
\text { Buturon }\end{array}$ & $\begin{array}{c}\text { HPLC-DAD } \\
\lambda=254 \mathrm{nmCenturysil} \mathrm{C18} \\
(250 \times 4.6 \mathrm{~mm}, 5 \mu \mathrm{m}) \\
\text { Eluent A: water; } \\
\text { Eluent } \mathrm{B}: \text { acetonitrile } \\
\text { A:B }(52: 48, v / v) \\
\text { Flow rate: } 1.0 \mathrm{~mL} / \mathrm{min}\end{array}$ & $\begin{array}{c}\text { Linearity: } 0.30 \div 150.0 \mathrm{ng} / \mathrm{mL} \text { for } \\
\text { soybean milk } \\
\text { Linearity: } 0.20 \div 150.0 \mathrm{ng} / \mathrm{g} \text { for tomato } \\
\text { LOD: } 0.10 \div 0.20 \mathrm{ng} / \mathrm{mL} \text { for } \\
\text { soybean milk } \\
\text { LOD: } 0.06 \div 0.15 \mathrm{ng} / \mathrm{g} \text { for tomato } \\
\text { Recovery: } 86.0 \div 115.2 \%\end{array}$ & [28] \\
\hline \multicolumn{4}{|c|}{ Phenyluracil derivatives } \\
\hline $\begin{array}{l}\text { Orange, apple, grape, mango, } \\
\text { banana, pear, peachTiafenacil } \\
\text { and its six metabolites }\end{array}$ & $\begin{array}{c}\text { UHPLC-TQ-ESI-MS/MS } \\
\text { Multiple reaction monitoring }(\mathrm{MRM}) \text { mode } \\
\text { Waters CORTECS C18 } \\
(150 \times 2.1 \mathrm{~mm}, 2.7 \mu \mathrm{m}) \\
\text { Eluent A: water containing } \\
0.1 \% \text { formic acid; } \\
\text { Eluent B: acetonitrile } \\
\text { Gradient elution } \\
\text { Flow rate: } 0.4 \mathrm{~mL} / \mathrm{min}\end{array}$ & $\begin{array}{l}\text { Linearity: } 5 \div 1000 \mu \mathrm{g} / \mathrm{kg} \\
\text { LOQ: } 10 \mu \mathrm{g} / \mathrm{kg} \\
\text { Intraday precision (RSD): } 1.0 \div 13.0 \% \\
\text { Interday precision (RSD): } 1.1 \div 14.6 \% \\
\text { Recovery: } 73 \div 105 \%\end{array}$ & [29] \\
\hline \multicolumn{4}{|c|}{ Multiclass pesticides } \\
\hline $\begin{array}{c}\text { Rice (Oryza sativa L.) } \\
\text { Multiclass pesticides (155) }\end{array}$ & $\begin{array}{c}\text { UHPLC-ESI-QTRAP-MS } \\
\text { Multiple reaction monitoring }(\mathrm{MRM}) \text { mode } \\
\text { Fusion-RP } 80 \mathrm{~A} \\
(50 \times 2 \mathrm{~mm}, 4 \mu \mathrm{m}) \\
\text { Eluent A: } 0.1 \% \text { formic acid } \\
\text { aqueous solution; } \\
\text { Eluent B: } 0.1 \% \text { formic acid in methanol } \\
\text { Gradient elution } \\
\text { Flow rate: } 0.25 \mathrm{~mL} / \mathrm{min}\end{array}$ & $\begin{array}{c}\text { Linearity: } 5 \div 50 \mu \mathrm{g} / \mathrm{L} \text { and } 5 \div 60 \mu \mathrm{g} / \mathrm{L} \\
\text { LOQ: } 5 \mu \mathrm{g} / \mathrm{kg} \\
\text { Recovery: } 77.1 \div 111.5 \%\end{array}$ & [30] \\
\hline $\begin{array}{c}\text { Pecan nuts } \\
\text { Multiclass pesticides (47) }\end{array}$ & $\begin{array}{c}\text { LC-TQ-ESI-MS/MS } \\
\text { Selected reaction monitoring }(\mathrm{SRM}) \text { mode } \\
\text { Pursuit XRs Ultra C18 } \\
(100 \times 2.0 \mathrm{~mm}, 1.7 \mu \mathrm{m}) \\
\text { Eluent A: aqueous } 5 \mathrm{mmol} / \mathrm{L} \\
\text { ammonium formate; } \\
\text { Eluent B: methanol } \\
\text { Gradient elution } \\
\text { Flow rate: } 0.150 \mathrm{~mL} / \mathrm{min}\end{array}$ & $\begin{array}{c}\text { Linearity: } 2.5 \div 125 \mu \mathrm{g} / \mathrm{L} \\
\text { LOD: } 2 \div 3 \mu \mathrm{g} / \mathrm{kg} \\
\text { Recovery: } 70 \div 120 \%\end{array}$ & [31] \\
\hline
\end{tabular}


Table 1. Cont.

\begin{tabular}{ccc}
\hline Matrix/Compound & Chromatographic Conditions & Other Parameters \\
& UPLC-TQ-ESI-MS/MS & \\
& Multiple reaction monitoring (MRM) mode & Linearity: not given \\
Sugarcane spirits & Acquity UPLC HSS T3 & LOD: $5 \mu \mathrm{g} / \mathrm{L}$ \\
(Brazilian cachaças) & $(150 \times 0.3 \mathrm{~mm}, 1.8 \mu \mathrm{m})$ & Accuracy: $80 \div 123 \%$ \\
Multiclass pesticides (10) & Eluent A: water; & Intraday precision (RSD): $0.31 \div 44.17 \%$ \\
& Eluent B: acetonitrile & Interday precision (RSD): $0.23 \div 22.78 \%$ \\
& Gradient elution & \\
\hline
\end{tabular}

\section{Other pesticides}

\section{LC-TQ-ESI-MS/MS}

Multiple reaction monitoring (MRM) mode

$$
\text { Chiralpak IG }
$$

$(250 \times 4.6 \mathrm{~mm}, 5 \mu \mathrm{m})$ with a Chiralpack IG guard column

$(10 \times 4 \mathrm{~mm}, 5 \mu \mathrm{m})$

Cucumber, tomato, cabbage, grape, mulberry, apple, pear

Chiral pesticides (22)
Eluent A: acetonitrile;

Eluent B: ultrapure water containing

$5 \mathrm{mmol} / \mathrm{L}$ ammonium acetate and

$0.1 \%$ formic acid

A:B $(65: 35, v / v)$

Flow rate: $0.6 \mathrm{~mL} / \mathrm{min}$

\author{
Linearity: $1 \div 200 \mathrm{ng} / \mathrm{g}$ \\ ILOQ (instrumental LOQ): \\ $0.33 \div 1.50 \mathrm{ng} / \mathrm{g}$ \\ MLOQ (method LOQ): $0.15 \div 1.00$ \\ ng/gRecovery: $84.0 \div 112.3 \%$ \\ Intraday precision (RSD): $2.3 \div 10.9 \%$ \\ Interday precision (RSD): $3.0 \div 11.2 \%$
}

LC-ESI-QTRAP-MS

Multiple reaction monitoring (MRM) mode

HILIC-column, Obelisc N

$(2.1 \times 150 \mathrm{~mm}, 5 \mu \mathrm{m})$

Eluent A: water with $1 \%$ formic acid;

Eluent B: acetonitrile

Gradient elution

Flow rate: $500 \mu \mathrm{L} / \mathrm{min}$

\author{
Linearity: $0.1 \div 100 \mathrm{ng} / \mathrm{mL}$ \\ LOQ: $0.02 \div 0.5 \mathrm{mg} / \mathrm{kg}$ \\ Recovery: $70 \div 120 \%$
}

Highly polar pesticides (14)

Non-steroidal anti-inflammatory compounds

(derivatives of phenylpropionic acid, phenylacetic acid and acetylsalicylic acid) and chloramphenicol

\begin{tabular}{|c|c|c|c|}
\hline $\begin{array}{c}\text { Bovine milk, } \\
\text { ovine milk } \\
\text { NSAIDs: Carprofen } \\
\text { (CPF), Tolfenamic acid (TFA), } \\
\text { 5-hydroxy flunixin (HFX), } \\
\text { Diclofenac (D), } \\
\text { 4-methylaminoantipyrin } \\
\text { (MAAP), } \\
\text { Meloxicam (MX), Ibuprofen } \\
\text { (I), Phenylbutazone (PBZ); } \\
\text { antibiotic: Chloramphenicol } \\
\text { (CHP) }\end{array}$ & $\begin{array}{c}\text { LC-ESI-QTRAP-MS } \\
\text { Scheduled multiple reaction monitoring } \\
(\mathrm{sMRM}) \text { mode } \\
\text { Kinetex XB-C18 } \\
(100 \times 2.1 \mathrm{~mm}, 2.6 \mu \mathrm{m}) \\
\text { Eluent A: water containing } \\
0.1 \% \text { formic acid; } \\
\text { Eluent B: methanol } \\
\text { Gradient elution } \\
\text { Flow rate: } 0.4 \mathrm{~mL} / \mathrm{min}\end{array}$ & $\begin{array}{c}\text { For all compounds:LOQ }(\mu \mathrm{g} / \mathrm{kg}) \text { : } \\
0.05 \text { (for D) } \\
0.15 \text { (for CHP) } \\
2.5(\text { for PBZ) } \\
5 \text { (for I) } \\
7.5 \text { (for MX) } \\
20 \text { (for HFX) } \\
25 \text { (for TFA, MAAP) } \\
250 \text { (for CPF) } \\
\text { Accuracy: } 87 \div 108 \% \\
\text { Interday precision }(\mathrm{CV}): 3 \div 16 \%\end{array}$ & [35] \\
\hline $\begin{array}{c}\text { Bovine milk } \\
\text { Diclofenac (D), } \\
\text { Flurbiprofen (FB), } \\
\text { Ketoprofen (KP), } \\
\text { Mefenamic acid (MA) }\end{array}$ & $\begin{array}{c}\text { HPLC-TQ-ESI-MS } / \mathrm{MS} \\
\text { Luna C18 } \\
(250 \times 2.0 \mathrm{~mm}, 5 \mu \mathrm{m}) \\
\text { Eluent A: methanol; } \\
\text { Eluent B: } 0.05 \% \text { aqueous solution of } \\
\text { formic acid } \\
\text { A:B }(3: 1, v / v) \\
\text { Flow rate: } 0.4 \mathrm{~mL} / \mathrm{min}\end{array}$ & $\begin{array}{c}\text { Linearity }(\mu \mathrm{g} / \mathrm{kg}): \\
0.03 \div 200(\text { for } \mathrm{D}, \mathrm{KP}) \\
0.03 \div 300(\text { for } \mathrm{FB}) \\
0.1 \div 250 \text { (for } \mathrm{MA}) \\
\text { LOD }(\mu \mathrm{g} / \mathrm{kg}): \\
0.01 \text { (for } \mathrm{D}, \mathrm{KP}, \mathrm{FB}) \\
0.03 \text { (for } \mathrm{MA}) \\
\text { Recovery: } 96 \div 107 \%\end{array}$ & [36] \\
\hline
\end{tabular}


Table 1. Cont.

\begin{tabular}{|c|c|c|c|}
\hline Matrix/Compound & Chromatographic Conditions & Other Parameters & Refs. \\
\hline $\begin{array}{l}\text { Meat of swine, chicken } \\
\text { and bovine } \\
\text { Multiclass NSAIDs (47) }\end{array}$ & $\begin{array}{c}\text { LC-TQ-ESI-MS/MS } \\
\text { Hypersil Gold C18 } \\
(150 \times 2.1 \mathrm{~mm}, 5 \mu \mathrm{m}) \\
\text { Eluent A: } 0.1 \% \text { formic acid with } \\
0.5 \mathrm{mmol} / \mathrm{L} \text { ammonium acetate; } \\
\text { Eluent B: acetonitrile }\end{array}$ & $\begin{array}{c}\text { Linearity: } 0.1 \div 50 \mathrm{ng} / \mathrm{mL} \\
\text { LOD: } 0.1 \div 0.5 \mathrm{ng} / \mathrm{g} \\
\text { Intraday precision (RSD): } 2.2 \div 5.6 \% \\
\text { Interday precision (RSD): } 5.3 \div 12.6 \% \\
\text { Recovery: } 72.4 \div 97.1 \%\end{array}$ & [37] \\
\hline $\begin{array}{c}\text { Bovine milk } \\
\text { Veterinary drugs: } \\
\text { Acetanilide (AAN), } \\
\text { Anthranilic acid (ANA), } \\
\text { Antipyrine (AP), } \\
\text { Cyproheptadine (CHD), } \\
\text { Diphenhydramine (DH), } \\
\text { DL-methylephedrine (ME), } \\
\text { Phenacetin (PA) }\end{array}$ & $\begin{array}{c}\text { LC-TQ-ESI-MS/MS } \\
\text { Multiple reaction monitoring }(\mathrm{MRM}) \text { mode } \\
\text { Waters Xbridge C18 } \\
(150 \times 2.1 \mathrm{~mm}, 3.5 \mu \mathrm{m}) \\
\text { Eluent A: } 0.1 \% \text { formic acid in water; } \\
\text { Eluent B: } 0.1 \% \text { formic acid in acetonitrile } \\
\text { Gradient elution } \\
\text { Flow rate: } 0.2 \mathrm{~mL} / \mathrm{min}\end{array}$ & $\begin{array}{c}\text { Linearity: } 1 \div 40 \mu \mathrm{g} / \mathrm{kg} \\
\text { LOD }(\mu \mathrm{g} / \mathrm{g}): \\
0.3(\text { for } \mathrm{AP}) \\
0.4 \text { (for CHD, ME) } \\
0.5 \text { (for DH) } \\
0.6 \text { (for PA) } \\
2.1 \text { (for AAN, ANA) } \\
\text { Recovery: } 71.2 \div 103.8 \% \\
\text { Intraday precision (RSD): } 0.7 \div 6.4 \% \\
\text { Interday precision (RSD): } 0.1 \div 8.6 \%\end{array}$ & [38] \\
\hline $\begin{array}{l}\text { Fish tissues } \\
\text { Ibuprofen, } \\
\text { Indoprofen, Pranoprofen, } \\
\text { Flurbiprofen, Ketoprofen, } \\
\text { Carprofen, } \\
\text { Naproxen, } \\
\text { Loxoprofen, } \\
\text { Etodolac }\end{array}$ & $\begin{array}{c}\text { UHPLC-TQ-ESI-MS/MS } \\
\text { Multiple reaction monitoring (MRM) mode } \\
\text { Chiralpak ID } \\
(250 \times 4.6 \mathrm{~mm}, 5 \mu \mathrm{m}) \text { with guard column } \\
(10 \times 4.6 \mathrm{~mm}, 5 \mu \mathrm{m}) \\
\text { Eluent A: } 40 \% \text { acetonitrile } \\
\text { Eluent B: water containing } \\
20 \mathrm{mM} \mathrm{HCOONH} 4 \\
\text { Gradient elution } \\
\text { Flow rate: } 0.6 \mathrm{~mL} / \mathrm{min}\end{array}$ & $\begin{array}{c}\text { Linearity: } 2 \div 400 \mathrm{ng} / \mathrm{g} \\
\text { LOD: } 1 \div 8 \mathrm{ng} / \mathrm{g} \\
\text { Recovery: } 82.6 \div 106.7 \% \\
\text { Intraday precision }(\mathrm{RSD}) \leq 8.2 \% \\
\text { Interday precision }(\mathrm{RSD}) \leq 8.2 \%\end{array}$ & {$[39]$} \\
\hline $\begin{array}{c}\text { Meat, egg } \\
\text { Ibuprofen (I), } \\
\text { Naproxen (N), } \\
\text { Diclofenac (D), } \\
\text { Carprofen (CPF), } \\
\text { Ketoprofen (KP), } \\
\text { Tolfenamic acid (TFA), } \\
\text { Salicylic acid (SA) }\end{array}$ & $\begin{array}{c}\text { UPLC-TQ-ESI-MS/MS } \\
\text { Multiple reaction monitoring }(\mathrm{MRM}) \text { mode } \\
\text { Acquity UPLC BEH C18 } \\
(50 \times 2.1 \mathrm{~mm}, 1.7 \mu \mathrm{m}) \\
\text { Eluent A: methanol; } \\
\text { Eluent B: water with } 0.1 \% \text { formic acid } \\
\text { Gradient elution } \\
\text { Flow rate: } 0.25 \mathrm{~mL} / \mathrm{min}\end{array}$ & $\begin{array}{c}\text { Linearity }(\mu \mathrm{g} / \mathrm{kg}) \text { : } \\
5 \div 1500 \text { (for D), } 10 \div 1500 \text { (for } \mathrm{N}) \\
20 \div 1500 \text { (for CPF, KP, SA) } \\
30 \div 1500 \text { (for TFA), } 40 \div 1500 \text { (for I) } \\
\text { LOD }(\mu \mathrm{g} / \mathrm{kg} \text { ): } \\
9.1 \div 12.2 \text { (for I), } 2.1 \div 2.4 \text { (for } \mathrm{N} \text { ) } \\
1.2 \div 1.4 \text { (for D), } 5.7 \div 6.0 \text { (for CPF, KP) } \\
7.5 \div 10.7 \text { (for TFA), } 4.5 \div 5.6 \text { (for SA) } \\
\text { Intraday precision (RSD): } 4.06 \div 16.01 \% \\
\text { Interday precision (RSD): } 2.74 \div 14.25 \% \\
\text { Recovery: } 85.18 \div 109.8 \%\end{array}$ & {$[40]$} \\
\hline \multicolumn{4}{|c|}{ Antibiotics (fluoroquinolones) } \\
\hline $\begin{array}{l}\text { Chicken meat } \\
\text { Beef meat } \\
\text { Feroxacin (FRX), } \\
\text { Ofloxacin (OF) }\end{array}$ & $\begin{array}{c}\text { HPLC-FLD } \\
\lambda=278 \mathrm{~nm} \text { and } \\
466 \mathrm{~nm} \\
\text { Luna C18 }(250 \times 4.6 \mathrm{~mm}, 5 \mu \mathrm{m}) \\
\text { Eluent A: Methanol } \\
\text { Eluent B: } 0.05 \mathrm{~mol} / \mathrm{L} \text { phosphate buffer } \\
(\mathrm{pH}=6.4) \\
\text { Gradient elution } \\
\text { Flow rate } 0.7 \mathrm{~mL} / \mathrm{min} \text { at } 30^{\circ} \mathrm{C}\end{array}$ & $\begin{array}{c}\text { For FRX: } \\
\text { Linearity: } 40 \div 4000 \mu \mathrm{g} / \mathrm{kg} \\
\text { LOD: } 15 \mu \mathrm{g} / \mathrm{kg}, \mathrm{LOQ}: 40 \mu \mathrm{g} / \mathrm{kg} \\
\text { Recovery: } 98 \div 108 \% \\
\text { For OF: } \\
\text { Linearity: } 30 \div 3000 \mu \mathrm{g} / \mathrm{kg} \\
\text { LOD: } 10 \mu \mathrm{g} / \mathrm{kg}, \mathrm{LOQ}: 30 \mu \mathrm{g} / \mathrm{kg} \\
\text { Recovery: } 100 \div 107 \%\end{array}$ & [41] \\
\hline
\end{tabular}


Table 1. Cont.

\begin{tabular}{|c|c|c|c|}
\hline Matrix/Compound & Chromatographic Conditions & Other Parameters & Refs. \\
\hline \multicolumn{4}{|c|}{ Steroid compounds } \\
\hline $\begin{array}{l}\text { Meat samples of different } \\
\text { categories (chicken, beef, } \\
\text { sheep, camels) } \\
\text { Some estrogens: estrone (E1), } \\
\text { 17ß-estradiol (E2), estriol (E3), } \\
\text { natural estrogens and } 17-\alpha \\
\text { ethinylestradiol (E4) } \\
\text { an exoestrogen }\end{array}$ & $\begin{array}{l}\text { HPLC-DAD, } \lambda=220 \mathrm{~nm} \\
\text { Symmetry C18 } \\
(4.6 \times 150 \mathrm{~mm}, 3.5 \mu \mathrm{m}) \\
\text { Eluent A: acetonitrile } \\
\text { Eluent B: water } \\
\text { A:B: }(50: 50, v / v) \\
\text { Flow rate: } 1 \mathrm{~mL} / \mathrm{min}\end{array}$ & $\begin{array}{c}\mathrm{LOD}(\mu \mathrm{g} / \mathrm{g}): \\
0.126 \text { (for E1, E2) } \\
0.094 \text { (for E3, E4) } \\
\text { LOQ ( } \mu \mathrm{g} / \mathrm{g}): \\
0.350 \text { (for E1, E2) } \\
0.188 \text { (for E3, E4) }\end{array}$ & [42] \\
\hline $\begin{array}{l}\text { Samples of chicken egg white } \\
\text { Corticosterone }\end{array}$ & $\begin{array}{c}\text { HPLC-MS/MS } \\
\text { Agilent Zorbax Eclipse Plus C18 } \\
(2.1 \times 100 \mathrm{~mm}, 1.8 \mu \mathrm{m}) \\
\text { Eluent A: } 0.1 \% \text { formic acid in water } \\
\text { Eluent B: acetonitrile- } 0.1 \% \text { formic acid } \\
\text { Gradient elution } \\
\text { Flow rate: } 0.4 \mathrm{~mL} / \mathrm{min}\end{array}$ & $\begin{array}{l}\text { LOQ: } 0.02 \mathrm{ng} / \mathrm{mL} \\
\text { Recovery: } 48.1 \%\end{array}$ & [43] \\
\hline $\begin{array}{l}\text { Samples of Antarctic krill } \\
\text { (Euphausia superba Dana) } \\
17 \text { Endogenous and } \\
\text { exogenous steroid hormones }\end{array}$ & $\begin{array}{c}\text { UHPLC-MS } \\
\text { Acchrom Unitary C18 } \\
(2.1 \times 150 \mathrm{~mm}, 5 \mu \mathrm{m}) \\
\text { Eluent A: water containing } \\
0.1 \% \text { formic acid } \\
\text { Eluent B: methanol } \\
\text { Gradient elution } \\
\text { Flow rate: } 0.2 \mathrm{~mL} / \mathrm{min}\end{array}$ & $\begin{array}{c}\text { LOD: } 2 \div 30 \mathrm{ng} / \mathrm{kg} \\
\text { LOQ: } 10 \div 100 \mathrm{ng} / \mathrm{kg} \\
\text { Recovery: } 75.4 \div 110.6 \%\end{array}$ & [44] \\
\hline \multicolumn{4}{|c|}{ Antioxidants (polyphenols and related compounds) } \\
\hline $\begin{array}{l}\text { Samples of various food } \\
\text { consumed in Malaysia, such } \\
\text { as chewing gum, noodle, } \\
\text { snacks, nut, chocolate, fruit } \\
\text { juices, coffee, oat, biscuit } \\
\text { Synthetic phenolic } \\
\text { antioxidants (SPAs): } \\
\text { propyl gallate, tert- } \\
\text { butylhydroquinone, butylated } \\
\text { hydroxyanisole, and } \\
\text { butylated hydroxytoluene }\end{array}$ & $\begin{array}{c}\text { HPLC-DAD, } \lambda=280 \mathrm{~nm} \\
\text { Agilent } \\
\text { ZORBAX Eclipse XDB } 5 \mu \mathrm{m} \mathrm{C18} \\
(150 \mathrm{~mm} \times 4.6 \mathrm{~mm}, 5 \mu \mathrm{m}) \\
\text { Eluent A: ultrapure water } \\
\text { Eluent B: acetonitrile } \\
\text { Gradient elution } \\
\text { Flow rate: } 2.0 \mathrm{~mL} / \mathrm{min}\end{array}$ & $\begin{array}{l}\text { Linearity: } 1 \div 300 \mathrm{mg} / \mathrm{L} \\
\text { LOD: } 0.02 \div 0.67 \mathrm{mg} / \mathrm{L} \text {, } \\
\text { LOQ: } 0.06 \div 2.03 \mathrm{mg} / \mathrm{L} \\
\text { Precision: } 0.15 \div 0.84 \% \\
\text { Recovery: } 80.4 \div 119.0 \%\end{array}$ & [45] \\
\hline $\begin{array}{l}\text { Milk samples from dairy cows } \\
\text { Quercetin }\end{array}$ & $\begin{array}{c}\text { UHPLC-MS } / \text { MS } \\
\text { ZORBAX SB-C18 } \\
(50 \times 2.1 \mathrm{~mm} \times 1.8 \mu \mathrm{m}) \\
\text { Eluent A: methanol } \\
\text { Eluent B: } 0.5 \% \text { formic acid } \\
\text { Gradient elution } \\
\text { Flow rate: } 0.5 \mathrm{~mL} / \mathrm{min}\end{array}$ & $\begin{array}{c}\text { LOQ: } 1.0 \mu \mathrm{g} / \mathrm{kg} \\
\text { Intraday precision: }<10 \% \\
\text { Interday precision: }<15 \% \\
\text { Repeatability: } 3 \div 7.2 \% \\
\text { Reproducibility: } 6.1 \div 12 \% \\
\text { Recovery: } 98 \%\end{array}$ & [46] \\
\hline $\begin{array}{l}\text { Samples of green coffee } \\
\text { produced company from } \\
\text { Skopje, Macedonia } \\
\text { Chlorogenic acid }\end{array}$ & $\begin{array}{c}\text { RP-HPLC-DAD } \\
\lambda=325 \mathrm{~nm} \\
\text { Poroshell } 120 \mathrm{EC}-\mathrm{C} 18 \\
(50 \times 3 \mathrm{~mm}, 2.7 \mu \mathrm{m}) \\
\text { Eluent A: acetonitrile } \\
\text { Eluent B: water with } 1 \% \text { phosphoric acid } \\
\text { A:B }(10: 90, v / v) \\
\text { Flow rate: } 1 \mathrm{~mL} / \mathrm{min}\end{array}$ & $\begin{array}{l}\text { Linearity: } 12.33 \div 143.50 \mu \mathrm{g} / \mathrm{mL} \\
\text { LOD: } 0.29 \mathrm{pg} \\
\text { LOQ: } 0.96 \mathrm{pg} \\
\text { Intraday precision } \\
\text { (RSD peak area): } 0.19 \% \\
\text { (RSD height): } 1.32 \% \\
\text { Recovery: } 97.87 \div 106.67 \%\end{array}$ & [47] \\
\hline
\end{tabular}


Table 1. Cont.

\begin{tabular}{|c|c|c|c|}
\hline Matrix/Compound & Chromatographic Conditions & Other Parameters & Refs. \\
\hline $\begin{array}{c}\text { Samples of commercially } \\
\text { available red wines } \\
\text { from Serbia } \\
16 \text { selected phenolic } \\
\text { compounds: gallic acid (GA), } \\
\text { p-hydroxybenzoic acid (HBA), } \\
\text { catechin (CAT), syringic acid } \\
\text { (SGA), trans-cinnamic acid } \\
\text { (TCA), hesperetin (HP), } \\
\text { naringenin (NG), vanillic acid } \\
\text { (VA), benzoic acid (BZA), } \\
\text { coumaric acid (CMA), } \\
\text { resveratrol (RV), chlorogenic } \\
\text { acid (CGA), caffeic acid (CFA), } \\
\text { rutin (RN), quercetin (Q), } \\
\text { kaempferol (KF) }\end{array}$ & $\begin{array}{c}\text { HPLC-DAD } \\
\lambda=280 \mathrm{~nm}(\mathrm{GA}, \mathrm{HBA}, \mathrm{CAT}, \mathrm{SGA}, \\
\text { TCA, HP, NG) } \\
\lambda=225 \mathrm{~nm}(\mathrm{VA}, \mathrm{BZA}, \mathrm{CMA}, \mathrm{RV}) \\
\lambda=360 \mathrm{~nm}(\mathrm{KF}) \\
\text { Poroshell } 120 \mathrm{EC}-\mathrm{C} 18 \\
(4.6 \times 100 \mathrm{~mm}, 2.7 \mu \mathrm{m}) \\
\text { Eluent A: distilled water with } \\
0.1 \% \text { glacial acetic acid } \\
\text { Eluent B: acetonitrile with } \\
0.1 \% \text { glacial acetic acid } \\
\text { Gradient elution } \\
\text { Flow rate: } 1.0 \mathrm{~mL} / \mathrm{min}\end{array}$ & $\begin{array}{c}\text { Linearity }(\mathrm{mg} / \mathrm{L}) \text { : } \\
2.5 \div 25 \text { (for CAT, VA) } \\
1.0 \div 25 \text { (for other compounds) } \\
\text { LOD }(\mathrm{mg} / \mathrm{L}): \\
0.03 \text { (for RV) } \div 0.62 \text { (for CAT) } \\
\text { LOQ }(\mathrm{mg} / \mathrm{L}) \text { : } \\
0.11 \text { (for RV, TCA) } \div 2.08 \text { (for CAT) } \\
\text { Recovery: } 96.5 \div 100.9 \%\end{array}$ & [48] \\
\hline
\end{tabular}

It is commonly known that the HPLC-UV (DAD) technique has a lower sensitivity compared to the LC-MS/MS. However, owing to the new SPE (solid phase extraction) systems consisting novel polymers as adsorbents e.g., porous organic polymer Car-DMB, Py-DMB HCP (heterocyclic hypercrosslinked polymer), HPLC analysis further allows the quantification of some pesticides in food samples at concentrations of $\mathrm{ng} / \mathrm{g}[25,28]$. As is shown in Table 1, many HPLC-MS/MS techniques with triple quadrupole (TQ), electrospray ionization (ESI) in multiple reaction monitoring (MRM) mode or selected reaction monitoring (SRM) mode have been mainly used for the determination of different kind of pesticides [16,18-21,27,31,33]. In addition, the HPLC-MS/MS methods with electrospray ionization (ESI) and quadrupole trap (QTRAP) in multiple reaction monitoring (MRM) mode have been also employed in the analysis of various pesticides $[23,24,30,34]$. Whereas UHPLC-Q Orbitrap-ESI-MS/MS has been applied for the determination of highly polar pesticides and contaminants (glyphosate, aminomethyl phosphonic acid (AMPA), phosphonic acid, fosetyl-Al, chlorate, and perchlorate) in processed fruits, vegetables, and infant foods [22].

Studies $[19,33]$ indicate that chiral LC-TQ-ESI-MS/MS and UPLC-TQ-ESI-MS/MS in MRM mode have been successfully applied to the simultaneous enantioselective determination of chiral pesticides in different vegetables and fruits. Martínez et al. [27] determined 27 acidic herbicides and 8 phytohormones in fruits and vegetables using UHPLC-TQ-ESI-MS/MS technique in the MRM mode.

Several papers created during the last two years [35-41] demonstrate the importance of different CLC procedures to determine selected veterinary drugs in animal food and food products belonging to various groups including non-steroidal anti-inflammatory agents (NSAIDS), some antibiotics, and others according to EU Commission Decision 2002/657/EC requirements [35] to guarantee food safety.

Whereas, LC-MS/MS methods with triple quadrupole (TQ), electrospray ionization (ESI) in multiple reaction monitoring (MRM) mode have been used for the determination of multiclass NSAIDs in meat of swine, chicken, eggs, and bovine $[37,38,40]$. Developed chiral UHPLC-TQ-ESI-MS/MS in MRM mode have been successfully applied to the simultaneous determination of four profens enantiomers including naproxen, carprofen, indoprofen, and flurbiprofen in fish tissues [39]. The obtained LODs and LOQs for each enantiomer ranged from 1 to $8 \mathrm{ng} / \mathrm{g}$ and 2 to $10 \mathrm{ng} / \mathrm{g}$, respectively [39].

Kurjogi et al. [49] applied an HPLC-UV for the detection of antibiotics in milk samples originating from the dairy herds located in India. Similarly, Dinh et al. [50] elaborated QuEChERS-LC-MS/MS clean up method with UHPLC-MS/MS for the analysis of sulfonamides and potentiators, macrolides, lincosamides, quinolones and fluoro- 
quinolones, nitrofurans, nitroimidazoles, chloramphenicol, triphenyl-methane dyes, teracyclines, and metabolites in cultured and wild seafood sold (in red-meat fish, white-meat fish, and shrimp).

Studies confirm the vital role of HPLC with diode array detection method and mass spectrometry for the analysis of some steroids in current residual food analysis of meat products and eggs coming from farmed animals, thus to control steroids in meat [42,43]. A reliable and sensitive UHPLC-MS method was also constructed by Han and Liu to detect 17 endogenous and exogenous steroid hormones including estrogens, androgens, glucocorticosteroids, and mineralocorticosteroids in Antarctic krill (Euphausia superba Dana) [44].

Another study shows the utility of HPLC with MS/MS based on the operation of a triple quadrupole (LC-ESI-MS/MS) for quality control of the species of meat or products by determining the presence of thermostable dipeptides (e.g., anserine, carnosine and balenin) [51].

Some studies demonstrate the important role of HPLC with UV, DAD, or FL detector as well as UHPLC-MS/MS in the study of patulin (mycotoxin) and related compounds in fruits e.g., mangoes, apples, grapes, oranges, and fruit products (juices and drinks) for children [52-58]. In this case C18 column and different usually binary mobile phases consisting, for example, of eluent A: $10 \mathrm{mM}$ ammonium acetate in water and eluent B: $10 \mathrm{mM}$ ammonium acetate in methanol [52] or acetonitrile-water [54] with gradient elution have been successfully applied. These methods allowed determining patulin at different levels given in $\mu \mathrm{g} / \mathrm{mL}$ or $\mu \mathrm{g} / \mathrm{kg}$ [52-58].

Several authors have also described the analytical methodologies based on HPLC to characterize the food composition i.e., to detect especially a new bioactive compounds with nutritional value and a proper biological activity, for example, antioxidant properties that are present in vegetables and fruits consumed in various countries. Developed methods are necessary to control the quality/authenticity of food and have been carried out by researchers during the last two years.

Numerous studies indicate that HPLC is the method of choice due to its precision and sensitivity for the determination and quantification of natural as well as synthetic antioxidants in various food/food products [45-47,59-64]. The main group of antioxidants investigated were phenolic compounds, especially phenolic acids, catechins, and flavonoids. Therefore the identification and assessment of antioxidant activity of different edible plant samples containing these bioactive compounds and their derivatives using highperformance liquid chromatography have been extensively investigated in the two last years. For example Yue et al. [45] developed and validated an HPLC-DAD method for the identification of selected synthetic phenolic antioxidants (SPAs) in chewing gum, noodle, snacks, nut, chocolate, fruit juices, coffee, oat, and biscuits. An interesting study performed by Cheung et al. [59] shows the utility of this technique for the determination of phenolic acids (16) and flavonoids (14) profiles in honey samples, thus for quality control of honey.

Gbylik-Sikorska et al. [46] developed for the first time an UHPLC-MS/MS method for the estimation of the pharmacokinetic parameters of quercetin in milk samples of dairy cows.

A few papers indicate the HPLC studies of different phenolic compounds in green coffee and the fruits of the three European plum cultivators [47,60].

Pepe et al. [61] undertook the study of the composition of polyphenols (26) and anthocyanins (12) found in Citrus sinensis and Vitis vinifera. RP-UHPLC-PDA combined with LCMS-IT-TOF (ion trap-time of flight mass spectrometer) was used in analysis of polyphenols and anthocyanins. HPLC with UV-Vis detection was also used for the determination of anthocyanin in skins and seeds of five Greek red grape varieties [62].

Similar study by means of HPLC-MS/MS method was performed to estimate the contents of some antioxidant components in grapevine seeds Vitis vinifera L cultivated in Italy [63]. The results of chromatographic analysis confirmed the presence of nine major flavonoids (apigenin, astragalin, hyperoside, isorhamnetin, kaempferol, myricetin, 
quercetin, quercitrin, and rutin) and two procyanidins (procyanidin $\mathrm{A}_{2}$ and procyanidin $\mathrm{B}$ ) in the studied extracts.

Carotenoids and polyphenols were evaluated and quantified by HPLC-DAD and UHPLC-Q-Orbitrap HRMS, respectively, in two-pigmented Lactuca sativa L. var. [64]. Separation and quantification of carotenoids were performed by HPLC-DAD on C18 column. Polyphenols analysis was performed by UHPLC-Q-Orbitrap HRMS on biphenyl column. LODs and LOQs of analyzed compounds were in the range of 0.03-0.05 and $0.10-0.16 \mathrm{ng} / \mathrm{g}$, respectively.

Another author Cirilli et al. [65] investigated iberin (an isothiocynate with chemoprevention of different tumors) in natural products and in different food supplements. Analysis was performed by UHPLC-PDA-ESI/MS. Three degradation products of iberin were identified, namely: thiourea, methyl thiocarbamate, and ethyl thiocarbamate. Similar study refers to 6-methoxymellein as the main ingredient responsible for the bitterness of carrot (Daucus carota L.) [66].

Summarizing, it can be stated that the studies described above confirm that validated high-performance liquid chromatography methods coupled with DAD, UV-Vis, MS/MS, and HPLC-TQ-ESI-MS/MS are the powerful tools in analysis i.e., separation, identification, and quantification of different natural and synthetic bioactive compounds occurring in food and food products for different purposes, i.e., authenticity and safety of food and food products.

It was stated that examined by column liquid chromatography bioactive compounds in food samples belonged to different chemical classes e.g., steroids, phenolic compounds, variety antibiotics (fluoroquinolones, tetracyclines, $\beta$-lactams), organophosphorus, phenyluracyl or triazines pesticides, and others. Therefore, both the factors, chemical diversity and the complexity of investigated mixtures, i.e., the kind of studied matrix were the biggest challenges in the case of HPLC technique and were accurately described in this review paper. A broad variety of packing material of column including a new one such as molecularly imprinted magnetic polymers as well as modern extraction systems like solid-phase extraction and salting-out extraction combined with switchable-hydrophilicity solvent liquid-liquid microextraction to sample preparation allow separation and quantification of new bioactive compounds like synthetic antioxidants or trace levels of different chemical groups of pesticides simultaneously (i.e., multiclass pesticides) in food. The use of chiral stationary phases improves the separation and determination of the selected stereoisomers (S- and R-form) of some imidazolinonen herbicides in food samples (e.g., soybean, peanut, wheat, maize, rice) and some NSAIDs belonging to profens i.e., ibuprofen, indoprofren, pranoprofen, flurbiprofen, ketoprofen, caprofen, naproxen and loxoprofen in fish tissues simultaneously at the level of $\mathrm{ng} / \mathrm{g}$.

Properly validated for optimal conditions HPLC method by means of DAD (PDA) and UV-Vis detector with gradient elution program makes this technique enough sensitive for the quantitative determination of different bioactive compounds including the selected pesticides and drugs in food samples in $\mu \mathrm{g} / \mathrm{mL}$ or $\mathrm{ng} / \mathrm{g}$, respectively.

\section{Gas Chromatography}

\section{GC in Analysis of Selected Bioactive Compounds in Food Samples}

Recent literature review shows that gas chromatography coupled to single or tandem mass spectrometric approaches (GC-MS, GC-MS/MS) served as an efficient tool for the determination of various organic compounds in food samples (Table 2). GC was used to quantify: 200 multiclass pesticides in fruits [67]; 14 lipophilic pesticides in raw propolis [68]; 5 organophosphorus pesticides (OPPs) in fruit juice and water [69], endocrine disrupting chemicals (EDCs) i.e., alkylphenols; 4 phenylphenols, bisphenol A; 7 parabens; 11 OPPs and triclosan in different cereal-based foodstuffs [70]; 4 isomers of hexachlorocyclohexane; 6 pyrethroid pesticides i.e., bifenthrin, fenpropathrin, cyhalothrin, cyfluthrin, cypermethrin, deltamethrin in milk [71]; 133 multiclass pesticides in pericarpium citri reticulatae (chenpi) [72]; 5 NSAIDs i.e., ibuprofen, paracetamol, diclofenac, naproxen, ketoprofen; 
3 natural estrogens i.e., estrone, 17 $\beta$-estradiol, estriol in Mussels Mytilus edulis trossulus [73], glyoxal and methylglyoxal in different alcoholic beverage and fermented foods [74], essential fatty acids in cereals and green vegetables [75], and fatty acids in grilled pork [76].

Table 2. GC in analysis of food samples.

\begin{tabular}{|c|c|c|c|}
\hline Matrix/Compound & Chromatographic Conditions & Other Parameters & Refs. \\
\hline \multicolumn{4}{|c|}{ Pesticides (organophosphorus and multiclass pesticides) } \\
\hline $\begin{array}{c}\text { Banana, watermelon, } \\
\text { pear, strawberry } \\
\text { Multiclass pesticides (200) }\end{array}$ & $\begin{array}{l}\text { GC-HRMS-Q-Orbitrap } \\
\text { Agilent VF-5 MS } \\
(30 \mathrm{~m} \times 0.25 \mathrm{~mm}, 0.25 \mu \mathrm{m}) \\
\text { Carrier gas: helium } \\
\text { Flow rate: } 1.0 \mathrm{~mL} / \mathrm{min}\end{array}$ & $\begin{array}{c}\text { Linearity: } 1 \div 100 \mu \mathrm{g} / \mathrm{kg} \\
\text { LOQ: } 5 \mu \mathrm{g} / \mathrm{kg} \\
\text { Recovery: } 70 \div 120 \% \\
\text { Intraday and Interday precision } \\
\text { (RSD): }<20 \%\end{array}$ & [67] \\
\hline $\begin{array}{c}\text { Raw propolis } \\
\text { Lipophilic pesticides (14) }\end{array}$ & $\begin{array}{c}\text { GC-EI-MS } / \text { MS } \\
\text { Multiple reaction monitoring } \\
\text { (MRM) mode } \\
\text { Agilent HP-5 MS } \\
(30 \mathrm{~m} \times 0.25 \mathrm{~mm}, 0.25 \mu \mathrm{m}) \\
\text { Carrier gas: helium } \\
\text { Flow rate: } 1.0 \mathrm{~mL} / \mathrm{min}\end{array}$ & $\begin{array}{l}\text { Linearity: } 0.001 \div 0.200 \mu \mathrm{g} / \mathrm{mL} \\
\text { LOQ: } 0.002 \div 0.020 \mu \mathrm{g} / \mathrm{g} \\
\text { Recovery: } 61 \div 106.8 \%\end{array}$ & {$[68]$} \\
\hline $\begin{array}{c}\text { Apple juice, grape juice, water } \\
\text { Organophosphorus } \\
\text { pesticides (OPPs): } \\
\text { Phorate (PHT), Dimethoate } \\
\text { (DMT), Diazinone (DZ), } \\
\text { Disulfoton (DSF), } \\
\text { Chlorpyrifos (CPF) }\end{array}$ & $\begin{array}{c}\text { GC-EI-MS } \\
\text { selected ion monitoring (SIM) mode } \\
\text { Agilent HP-5 MS } \\
(30 \mathrm{~m} \times 0.25 \mathrm{~mm}, 0.25 \mu \mathrm{m}) \\
\text { Carrier gas: helium } \\
\text { Flow rate: } 1.0 \mathrm{~mL} / \mathrm{min}\end{array}$ & $\begin{array}{c}\text { Linearity: } 2.0 \div 500.0 \mu \mathrm{g} / \mathrm{L} \\
\text { LOD }(\mu \mathrm{g} / \mathrm{L}): \\
0.9 \text { (for PHT), } 0.4 \text { (for DMT), } \\
0.6 \text { (for DZ), } 0.3 \text { (for DSF), } \\
1.0 \text { (for CPF) } \\
\text { Recovery: } 83 \div 105 \%\end{array}$ & [69] \\
\hline $\begin{array}{l}\text { Wheat flour, rice, spaghetti, } \\
\text { cheese tortellini, macaroni, } \\
\text { noodles, sesame regañas, wheat } \\
\text { tortillas, corn flakes, crunchy } \\
\text { fruit muesli, cookies, white } \\
\text { bread, multiseed } \\
\text { EDCs (Endocrine Disrupting } \\
\text { Chemicals) (24): } \\
\text { alkylphenols and } \\
\text { phenylphenols (4), } \\
\text { bisphenol A, parabens (7), } \\
\text { pesticides (11), } \\
\text { triclosan (personal care product) }\end{array}$ & $\begin{array}{c}\text { GC-EI-MS } \\
\text { selected ion monitoring (SIM) mode } \\
\text { DB- } 5 \mathrm{MS} \\
(30 \mathrm{~m} \times 0.25 \mathrm{~mm}, 0.25 \mu \mathrm{m}) \\
\text { Carrier gas: helium } \\
\text { Flow rate: } 1.0 \mathrm{~mL} / \mathrm{min}\end{array}$ & $\begin{array}{c}\text { For all compounds: } \\
\text { Linearity: } 1.3 \div 2500 \mathrm{ng} / \mathrm{kg} \\
\text { LOD: } 0.4 \div 23 \mathrm{ng} / \mathrm{kg} \\
\text { Intraday precision (RSD): } 3.8 \div 6.2 \% \\
\text { Interday precision (RSD): } 5.2 \div 7.2 \% \\
\text { Recovery: } 82 \div 105 \% \\
\text { For pesticides: } \\
\text { Linearity: } 21 \div 2500 \mathrm{ng} / \mathrm{kg} \\
\text { LOD: } 6.2 \div 23 \mathrm{ng} / \mathrm{kg} \\
\text { Intraday precision (RSD): } 5.0 \div 6.2 \% \\
\text { Interday precision (RSD): } 6.5 \div 7.2 \% \\
\text { Recovery: } 83 \div 105 \%\end{array}$ & [70] \\
\hline
\end{tabular}

\begin{tabular}{|c|c|c|c|}
\hline $\begin{array}{c}\text { Milk } \\
\text { Isomers of } \\
\text { hexachlorocyclohexane } \\
(\alpha-\mathrm{HCH}, \beta-\mathrm{HCH}, \gamma-\mathrm{HCH}, \\
\delta-\mathrm{HCH}) \text { and pyrethroid } \\
\text { pesticides (bifenthrin, } \\
\text { fenpropathrin, cyhalothrin, } \\
\text { cyfluthrin, cypermethrin, } \\
\text { deltamethrin) }\end{array}$ & $\begin{array}{c}\text { GC-ECD } \\
\text { ZB-5 } \\
(30 \mathrm{~m} \times 0.25 \mathrm{~mm}, 0.25 \mu \mathrm{m}) \\
\text { Carrier gas: nitrogen } \\
\text { Flow rate: } 0.72 \mathrm{~mL} / \mathrm{min}\end{array}$ & $\begin{array}{l}\text { For all compounds: } \\
\text { Linearity: } 0.00143 \div 3.57 \mathrm{mg} / \mathrm{L} \\
\text { LOD: } 0.07 \div 2 \mu \mathrm{g} / \mathrm{kg} \\
\text { LOQ: } 0.2 \div 5 \mu \mathrm{g} / \mathrm{kg} \\
\text { Recovery: } 70.1 \div 106.3 \%\end{array}$ & [71] \\
\hline $\begin{array}{c}\text { Pericarpium citri } \\
\text { reticulatae (chenpi) } \\
\text { Multiclass pesticides (133) }\end{array}$ & $\begin{array}{c}\text { GC-EI-MS/MS } \\
\text { Multiple reaction monitoring } \\
\text { (MRM) mode } \\
\text { DB-5MS IU } \\
(30 \mathrm{~m} \times 0.25 \mathrm{~mm}, 0.25 \mu \mathrm{m}) \\
\text { Carrier gas: helium } \\
\text { Flow rate: } 1.5 \mathrm{~mL} / \mathrm{min}\end{array}$ & $\begin{array}{c}\text { Linearity: } 1 \div 200 \mathrm{ng} / \mathrm{mL} \\
\text { LOQ: } 0.005 \div 0.01 \mathrm{mg} / \mathrm{kg} \\
\text { Recovery: } 70 \div 112.2 \%\end{array}$ & [72] \\
\hline
\end{tabular}


Table 2. Cont.

\begin{tabular}{ccc}
\hline Matrix/Compound & Chromatographic Conditions & Other Parameters \\
\hline & Non-steroidal anti-inflammatory compounds (profens) and Steroids \\
\hline & & For all compounds: \\
& & LOD: $1 \div 7 \mathrm{ng} / \mathrm{g}$ \\
& & Intermediate precision (RSD): \\
& GC-MS & $0.24 \div 9.82 \%$ \\
Mussels Mytilus edulis trossulus & Selected ion monitoring (SIM) mode & Repeatability (RSD): $0.94 \div 7.82 \%$ \\
NSAID (5): ibuprofen, & Zebron ZB-5MSi & Recovery: $80 \div 118 \%$ \\
paracetamol, diclofenac, & Carrier gas: helium & For NSAID: \\
naproxen, ketoprofen & & LOD: $1 \div 2$ ng $/ \mathrm{g}$ \\
Natural estrogens (3): & & Intermediate precision (RSD): \\
estrone, $17 \beta$-estradiol, estriol & & $0.69 \div 7.85 \%$ \\
& & Repeatability (RSD): $0.94 \div 4.92 \%$ \\
& & Recovery: $80 \div 115 \%$
\end{tabular}

\section{Fatty acids}

Cereals and green vegetables Essential fatty acids
ID-GC/MS

HP-88 capillary column $(60 \mathrm{~m} \times 0.25 \mathrm{~mm}, 0.2 \mu \mathrm{m})$

Carrier gas: helium

Flow rate: $1.0 \mathrm{~mL} / \mathrm{min}$

Repeatability (RSD):

$0.23 \div 1.61 \%$ for the cereal samples

$0.39 \div 1.89 \%$ for vegetable samples

Repeatability for linoleic acid (RSD): 1.48 and $0.95 \%$ for rice and wheat flours

Content of linoleic acid:

$3614 \mathrm{mg} / \mathrm{kg}$ for rice flour

$8402 \mathrm{mg} / \mathrm{kg}$ for wheat flour

$6353 \mathrm{mg} / \mathrm{kg}$ for spinach powder

$1353 \mathrm{mg} / \mathrm{kg}$ for Kimchi cabbage powder;

Content of $\alpha$-linolenic acid:

$19786 \mathrm{mg} / \mathrm{kg}$ for spinach powder

$9533 \mathrm{mg} / \mathrm{kg}$ for Kimchi cabbage powder

LOQ: $0.1 \%$ of the total fatty acids Content of:

Palmitic acid: $17.3 \div 55.4 \%$

Stearic acid: $8.8 \div 20.9 \%$

Oleic acid: $24.4 \div 48.8 \%$

Linoleic acid: $0.5 \div 3.6 \%$

Fatty acids

GC-MS

CP-Sil88

$(100 \mathrm{~m} \times 0.25 \mathrm{~mm}, 0.2 \mu \mathrm{m})$

Carrier gas: helium
Stearidonic acid: $<0.1 \div 4.2 \%$

Docosahexaenoic acid: $0.5 \div 1.4 \%$

Gamma linolenic acid: $<1 \%$

di-homo- $\gamma$ - linolenic acid: $<1 \%$

eicosapentaenoic acid: $<1 \%$

\section{Other compounds}

Alcoholic beverage (wine, bear, makgeoli, soju, and fruit liquor)

Fermented foods (soybean

paste, red pepper paste, soy sauce)

Glyoxal (GX),

Methylglyoxal (MGX)

\section{GC-MS}

HP-InnoWax capillary column $(60 \mathrm{~m} \times 0.25 \mathrm{~mm}, 0.25 \mu \mathrm{m})$

Carrier gas: helium

Flow rate: $1.0 \mathrm{~mL} / \mathrm{min}$

\section{For GLX:}

Working range $5 \div 4000 \mu \mathrm{g} / \mathrm{kg}$ Accuracy: $93.3 \div 104.5 \%$

Intraday precision: $4.3 \div 7.6 \%$ Interday precision: $3.0 \div 6.4 \%$ LOD: $1.1 \mu \mathrm{g} / \mathrm{kg}$

For MGX:

Working range $5 \div 4000 \mu \mathrm{g} / \mathrm{kg}$ Accuracy: $92.9 \div 104.2 \%$ Intraday precision: $4.8 \div 7.9 \%$ Interday precision: $3.6 \div 7.5 \%$ LOD: $0.7 \mu \mathrm{g} / \mathrm{kg}$ 
Crude fat, total saturated acids, and total trans fatty acids in home meal replacements, and restaurant foods were analyzed using GC-FID (gas chromatography-flame ionization detector). Total crude fat contents were $0.61 \div 6.75 \mathrm{~g} / 100 \mathrm{~g}$, and $0.22 \div 5.69 \mathrm{~g} / 100 \mathrm{~g}$ for home meal replacements and restaurant foods, respectively. Total saturated fatty acids contents were $0.08 \div 1.42 \mathrm{~g} / 100 \mathrm{~g}$, and $0.07 \div 1.44 \mathrm{~g} / 100 \mathrm{~g}$ for home meal replacements and restaurant foods, respectively. Total trans fatty acids contents were $0.0 \div 0.11 \mathrm{~g} / 100 \mathrm{~g}$, and $0.0 \div 0.07 \mathrm{~g} / 100 \mathrm{~g}$ for home meal replacements and restaurant foods, respectively [77]. Fatty acids in the form of methyl esters were also determined using the GC-FID technique in four bee products. The authors of the study compared the total fatty acid concentration (saturated, unsaturated, omega-3, omega-6, the ratio of saturated and unsaturated, omega3/omega-6 fatty acids and trans fatty acids) [78]. Fruehwirth et al. [79] investigated the lipid oxidation in stored margarine using GC-FID method. Volatile components and fatty acids present in margarines were tested. Acetone and hexanal increased in all types of margarine during storage.

Study [80] shows the applicability of GC-MS analysis for identification of chemical components with different activity including antioxidant properties of varieties, not well described in literature, of edible plants and fruits cultivated in different countries. GC-MS was successfully applied for the separation and identification of chemical components with antioxidant activity such as different phenolic acids from citrus fruits cultivated in India i.e., grapefruits. The major components found were: limonene, methyl-cyclohexane, hexane-3one, 3-hexanol, 2-hexanol, myrcene, sabinene, nonanal, neral, geranyl acetate, ostole. These compounds might contribute to the antioxidant activity of the juice and oil [80].

The reviewed papers confirm that gas chromatography has recently been used to study food and edible plants (the contents of pesticides, endocrine disrupting chemicals, NSAIDs, natural estrogens, glyoxal, methylglyoxal, fatty acids, compounds with antioxidant properties, such as e.g., flavonoids, phenolic compounds). The most commonly used gas chromatography was combined with a mass spectrometer or a dual mass spectrometer with electrospray ionization (GC-EI-MS, GC-EI-MS/MS). The presented papers show the utility of this technique for both, i.e., residue analysis of multiclass pesticides and NSAIDs simultaneously in food and food products as well as for the determination of new antibacterial and antitumor agents in edible plants.

\section{Combined Techniques}

In many cases, not one but two or more analytical techniques are required for determining the active substances present in food matrices. Nowadays, these combined techniques are powerful analytical tools with many applications. Several papers reported their utility in food analysis [81-86].

Carotenoids, phenolic compounds, and fatty acids were determined in tomato seed oil derived from cold break and hot break processing lines [81]. HPLC-DAD-ESI-MS on C18 column and two mobile phases in the gradient elution were used in the investigation of phenolic compounds. HPLC-DAD on C18 column and two mobile phases were used for the quantitative and qualitative analysis of carotenoids. Fatty acid profile was determined by GC-MS. Higher levels of carotenoids (lutein, lycopene, $\beta$-carotene) and phenolic compounds ((caffeic acid-glucoside isomer (CG), caffeic acid (CA), syringic acid (SyA), di-caffeoylquinic acid (di-CQA), and tri-Caffeoylquinic acid (tri-CQA)) were found in the cold pressed oil. The following fatty acids were the most abundant in the oil: linoleic acid, oleic acid, and palmitic acid [81].

Migas et al. [82] determined lutein and lutein mixed with zeaxanthin in eight dietary supplements. BMD-TLC (bivariant multiple development thin layer chromatography) was used for the analysis of lutein, $\beta$-carotene in samples. HPLC-DAD-ESI-MS was used for the isolation and identification of mixture of lutein and zeaxanthin. The proposed method was linear in the range $90 \div 500 \mathrm{ng} /$ point. Limits of detection and quantification were $50 \mathrm{ng} /$ point and $90 \mathrm{ng} /$ point, respectively. Method was precise, accurate, and robust. 
TLC was used for monitoring the formation of $\gamma$-aminobutyric acid (GABA) in traditional Indonesian foods fermented with thirty strains of lactic acid. For this purpose, silica gel $60 \mathrm{~F}_{254}$ plates and $n$-butanol-acetic acid-distilled water (5:2:2) mobile phase were used. On the other hand, for the quantitative determination of GABA, UPLC was used with the C18 column [83].

Aflatoxins are produced by fungi, including those on spoiled food. TLC on silica gel 60 plates using acetonitrile-methanol-trifluoroacetic acid (9:1:0.2, v/v/v) mobile phase and with the visualization using vanillin, $\mathrm{p}$-anisaldehyde solutions, or iodine vapor was a simple, robust, and non-quantitative method for the detection of aflatoxins. HPLC-DAD $(\lambda=200 \div 410 \mathrm{~nm}$ ) with C18 column and two eluents in gradient elution were used for the quantitative determination of aflatoxins. TOF/Q-TOF MS/MS was used for the detection of aflatoxin metabolites, and the sixteen possible metabolites were identified [84].

A novel and highly sensitive metastable state nanoparticle-enhanced Raman spectroscopy combined with thin layer chromatography (TLC-MSNERS) has been successfully used for the determination of pesticides such as thiabendazole, phosmet, and triazophos on fruit skin. An amphiphilic polymer polyurethane-Ag nanoparticle (AgNPs) has been employed as the MSNERS substrate [85]. Another work developed and validated a modified QuEChERS method to determine multiclass pesticides (207) in honey samples using both LC-MS/MS (154 compounds) and GC-MS/MS (53 compounds) [86].

In summary, the necessity to analyze samples with a complicated composition requires the use of combined techniques. Sometimes the matrix is so complex (it contains chemical compounds belonging to different chemical classes) that there is a need to use at least two analytical techniques to determine the composition of the analyzed sample. The reliability requirements of the analytical results often preclude the possibility of identifying the analytes solely on the basis of the retention time. Only the combination of the ability to separate complex mixtures using chromatographic methods with structural information (HPTLC/MS, LC/MS, GC/MS) enables reliable identification of food constituents.

Owing to the use of combined techniques, it is possible to significantly speed up and reduce the cost of analyzing due to less requirements for the stage of sample preparation for analysis.

The advantages of the combined techniques in food analysis are: the ability to identify unknown food constituents, information about their molecular weight and/or structure, easy detection of the overlap between peaks, and faster end results. In contrast, the disadvantages of the combined techniques are high investment costs.

\section{Conclusions}

The reviewed papers confirm that of all chromatographic techniques, liquid chromatography (LC) is the most universal technique that enables successful analysis of complex matrices including food products. The current high-performance liquid chromatography systems are crucial to assess the quality of food. HPLC method in combination with various detection modes i.e., HPLC-UV, HPLC-DAD(PDA) and HPLC-MS or HPLCMS/MS, respectively is selective, sensitive, accurate, and robust for the simultaneous determination of natural and synthetic bioactive molecules belonging to different chemical classes in complex food samples as residue of food production such as multiclass pesticides, NSAIDs or steroids, as well as a new food constituents (e.g., antioxidants) in edible plants cultivated in different countries. The use of modern spectroscopic techniques such as MS as detection system allows the identification and accurate study of the structure of all components occurring in food matrices.

While thin-layer chromatography coupled to densitometry and mass spectrometry could be the most suitable technique for preliminary screening and determination the antioxidant properties (TLC-DPPH) of food components. 
Gas chromatographic methods (GC-EI-MS, GC-EI-MS/MS) are also essential for the screening of different bioactive compounds including the pesticides and fatty acids in edible plants and in food products. Pesticides profiling in food samples done by HPLC and GC in combination with prior sample separation by means of modern microextraction systems can be valuable in rapid quality control of food and ensures food use safety.

Author Contributions: W.P., M.D. and A.P.-P. have collected the data, designed, and written the manuscript; A.P.-P., W.P. and M.D., have revised the manuscript. All authors have read and agreed to the published version of the manuscript.

Funding: This research received no external funding.

Institutional Review Board Statement: Not applicable.

Informed Consent Statement: Not applicable.

Data Availability Statement: Not applicable.

Conflicts of Interest: The authors declare that there is no conflict of interests regarding the publication of this paper.

\section{References}

1. Guaadaoui, A.; Benaicha, S.; Elmajdoub, N.; Bellaoui, M.; Hamal, A. What is a bioactive compound? A combined definition for a preliminary consensus. Int. J. Food Sci. Nutr. 2014, 3, 174-179. [CrossRef]

2. Anuuryanti, F.; Isnaeni, I.; Darmawati, A.; Rosyidah, I.; Dwiana, N. Method validation of contact and immersion TLC bioautography for determination of streptomycin sulfate in shrimp. Turk J. Pharm. Sci. 2020, 17, 254-258. [CrossRef] [PubMed]

3. Foudah, A.I.; Alam, P.; Abdel-Kader, M.S.; Shakeel, F.; Alqasoumi, S.I.; Salkini, A.M.; Yusufoglu, H.S. High-performance thin-layer chromatographic determination of trigonelline content in various extracts and different varieties of some commercial coffees available in the Saudi Arabian market. J. Planar Chromatogr. Mod. TLC 2020, 33, 43-50. [CrossRef]

4. Khairul, I.M.; Sostaric, T.; Lim, L.Y.; Hammer, K.; Locher, C. Development and validation of an HPTLC-DPPH assay and its application to the analysis of honey. J. Planar Chromatogr. Mod. TLC 2020, 33, 301-311.

5. Madhukar, N.S.; Vinayak, S.M. A novel digitally optimized rapid quantification of carcinogenic aryl azo amines from various food matrices by HPTLC-MS. J. Liq. Chromatogr. Rel. Technol. 2020, 43, 445-454. [CrossRef]

6. Piszcz, P.; Tomaszewska, M.; Głód, B.K. Estimation of the total antioxidant potential in the meat samples using thin-layer chromatography. Open Chem. 2020, 18, 50-57. [CrossRef]

7. Turkmen, Z.; Kurada, O. Rapid HPTLC determination of patulin in fruit-based baby food in Turkey. J. Planar Chromatogr. Mod. TLC 2020, 33, 209-217. [CrossRef]

8. Dąbrowska, M.; Sokalska, K.; Gumułka, P.; Binert-Kusztal, Ż.; Starek, M. Quantification of omega-3-fatty acids in dietary supplements and cooking products available on the polish market by thin-layer chromatography-densitometry. J. Planar Chromatogr. Mod. TLC 2019, 32, 13-24. [CrossRef]

9. Pawar, U.D.; Pawar, C.D.; Kulkarni, U.K.; Pardeshi, R.K.; Farooqui, M.; Shinde, D.B. Use of diphenylamine reagent for highperformance thin-layer chromatographic detection of organochloro insecticide endosulfan in biological samples. J. Planar Chromatogr. Mod. TLC 2019, 32, 65-68. [CrossRef]

10. Patil, A.S.; Patil, K.P.; Patil, A.B.; Kulkarni, P.M.; Chandegaonkar, V.R.; More, B.P.; Mane, D.V. A new chromogenic spray reagent for the detection and identification of oxyfluorten herbicide in biological material by high-performance thin-layer chromatography. J. Planar Chromatogr. Mod. TLC 2019, 32, 69-71. [CrossRef]

11. Pawar, U.D.; Pawar, C.D.; Kulkarni, U.K.; Pardeshi, R.K.; Farooqui, M.; Shinde, D.B. New chromogenic reagent for highperformance thin-layer chromatographic detection of organophosphorus insecticide monocrotophos in biological materials. $J$. Planar Chromatogr. Mod. TLC 2019, 32, 61-64. [CrossRef]

12. Patil, K.P.; Patil, A.S.; Patil, A.B.; Kulkarni, P.M.; Chandegaonkar, V.R.; More, B.P. A new chromogenic spray reagent for the detection and identification of 2,4-dichlorophenol, an intermediate of 2,4-D herbicide in biological material by high-performance thin-layer chromatography. J. Planar Chromatogr. Mod. TLC 2019, 32, 431-434. [CrossRef]

13. Pawar, U.D.; Pawar, C.D.; Kulkarni, U.K.; Pardeshi, R.K. Development method of high-performance thin-layer chromatographic detection of synthetic organophosphate insecticide profenofos in visceral samples. J. Planar Chromatogr. Mod. TLC 2020, 33, 203-206. [CrossRef]

14. Pawar, U.D.; Pawar, C.D.; Mavie, R.R.; Pardeshi, R.K. Development of a new chromogenic reagent for the detection of organophosphorus herbicide glyphosate in biological samples. J. Planar Chromatogr. Mod. TLC 2019, 32, 435-437. [CrossRef]

15. Hussain, M.; Aftab, K.; Iqbal, M.; Ali, S.; Rizwan, M.; Alkahtani, S.; Abdel-Daim, M.M. Determination of pesticide residue in brinjal sample using HPTLC and developing a cost-effective method alternative to HPLC. J. Chem. 2020, 8180320. [CrossRef] 
16. Zheng, W.; Choi, J.M.; Abd El-Aty, A.M.; Yoo, K.H.; Park, D.H.; Kim, S.K.; Kang, Y.S.; Hacımüftüoğlu, A.; Wang, J.; Shim, J.H.; et al. Simultaneous determination of spinosad, temephos, and piperonyl butoxide in animal-derived food using LC-MS/MS. Biomed. Chromatogr. 2019, 33, e4493. [CrossRef] [PubMed]

17. Huang, X.C.; Ma, J.K.; Feng, R.X.; Wei, S.L. Simultaneous determination of five organophosphorus pesticide residues in different food samples by solid-phase microextraction fibers coupled with high-performance liquid chromatography. J. Sci. Food Agric. 2019, 99, 6998-7007. [CrossRef] [PubMed]

18. Guo, T.; Wang, X.; Wang, H.; Hu, Y.; Zhang, S.; Zhao, R. Determination of phenoxy acid herbicides in cereals using highperformance liquid chromatography-tandem mass spectrometry. J. Food Prot. 2019, 82, 1160-1165. [CrossRef] [PubMed]

19. Li, R.; Hu, M.; Liu, K.; Zhang, H.; Li, X.; Tan, H. Trace enantioselective determination of imidazolinone herbicides in various food matrices using a modified QuEChERS method and ultra-performance liquid chromatography/tandem mass spectrometry. Food Anal. Methods 2019, 12, 2647-2664. [CrossRef]

20. Tan, S.; Yu, H.; He, Y.; Wang, M.; Liu, G.; Hong, S.; Yan, F.; Wang, Y.; Wang, M.; Li, T.; et al. A dummy molecularly imprinted solid-phase extraction coupled with liquid chromatography-tandem mass spectrometry for selective determination of four pyridine carboxylic acid herbicides in milk. J. Chromatogr. B 2019, 1108, 65-72. [CrossRef]

21. Francesquett, J.Z.; Rizzetti, T.M.; Cadaval, T.R.S., Jr.; Prestes, O.D.; Adaime, M.B.; Zanella, R. Simultaneous determination of the quaternary ammonium pesticides paraquat, diquat, chlormequat, and mepiquat in barley and wheat using a modified quick polar pesticides method, diluted standard addition calibration and hydrophilic interaction liquid chromatography coupled to tandem mass spectrometry. J. Chromatogr. A 2019, 1592, 101-111.

22. Savini, S.; Bandini, M.; Sannino, A. An improved, rapid, and sensitive ultra-high-performance liquid chromatography-highresolution orbitrap mass spectrometry analysis for the determination of highly polar pesticides and contaminants in processed fruits and vegetables. J. Agric. Food Chem. 2019, 67, 2716-2722. [CrossRef]

23. Zhang, Y.; Dang, Y.; Lin, X.; An, K.; Li, J.; Zhang, M. Determination of glyphosate and glufosinate in corn using multi-walled carbon nanotubes followed by ultra high performance liquid chromatography coupled with tandem mass spectrometry. $J$. Chromatogr. A 2020, 1619, 460939. [CrossRef]

24. Lopez, S.H.; Dias, J.; Mol, H.; de Kok, A. Selective multiresidue determination of highy polar anionic pesticides in plantbased milk, wine and beer using hydrophilic interaction liquid chromatography combined with tandem mass spectrometry. $J$. Chromatogr. A 2020, 1625, 461226. [CrossRef]

25. Li, G.; Meng, X.; Wang, J.; Wang, Q.; Zhou, J.; Wang, C.; Wu, Q.; Wang, Z. A low-cost and high-efficiency carbazole-based porous organic polymer as a novel sorbent for solid-phase extraction of triazine herbicides in vegetables. Food Chem. 2020, $309,125618$. [CrossRef] [PubMed]

26. Zhang, L.; Liu, J.; Wang, C.; Yu, R. Silica gel immobilized ionic liquid dispersion extraction and separation of triazine and acetanilide herbicides in beans. Food Anal. Methods 2020, 13, 1791-1798. [CrossRef]

27. Martínez, Á.G.; Arrebola Liébanas, F.J.; Valverde, R.S.; Hernández Torres, M.E.; Casinello, J.R.; Garrido Frenich, A. Multifamily determination of phytohormones and acidic herbicides in fruits and vegetables by liquid chromatography-tandem mass spectrometry under accredited conditions. Foods 2020, 9, 906. [CrossRef]

28. Wang, Q.; Wang, C.; Wang, J.; Liu, W.; Hao, L.; Zhou, J.; Wang, Z.; Wu, Q. Sensitive determination of phenylurea herbicides in soybean milk and tomato samples by a novel hypercrosslinked polymer based solid-phase extraction coupled with high performance liquid chromatography. Food Chem. 2020, 317, 126410. [CrossRef] [PubMed]

29. Hu, M.; Tan, H.; Li, Y.; Qiu, J.; Liu, L.; Zeng, D. Simultaneous determination of tiafenacil and its six metabolites in fruits using ultra-high-performance liquid chromatography/tandem mass spectrometry. Food Chem. 2020, 327, 127015. [CrossRef]

30. Melo, M.G.; Carqueijo, A.; Freitas, A.; Barbosa, J.; Silva, A.S. Modified QuEChERS extraction and HPLC-MS/MS for simultaneous determination of 155 pesticide residues in rice (Oryza sativa L.). Foods 2020, 9, 18. [CrossRef]

31. Barci, P.E.P.; Alves, L.S.; Avellar, A.A.S.; Cendon, L.R.; dos Santos, P.J.; Stringhini, F.M.; Prestes, O.D.; Zanella, R. Modified QuEChERS method for multiresidue determination of pesticides in pecan nuts by liquid chromatography tandem mass spectrometry. Food Anal. Methods 2020, 13, 793-801. [CrossRef]

32. Pereira dos Santos, N.G.; Maciel, E.V.S.; Mejía-Carmona, K.; Lanças, F.M. Multidimensional capillary liquid chromatographytandem mass spectrometry for the determination of multiclass pesticides in "sugarcane spirits" (cachaças). Anal. Bioanal. Chem. 2020, 412, 7789-7797. [CrossRef]

33. Zhao, P.; Wang, Z.; Gao, X.; Guo, X.; Zhao, L. Simultaneous enantioselective determination of 22 chiral pesticides in fruits and vegetables using chiral liquid chromatography coupled with tandem mass spectrometry. Food Chem. 2019, 277, 298-306. [CrossRef]

34. López, S.H.; Scholten, J.; Kiedrowska, B.; de Kok, A. Method validation and application of a selective multiresidue analysis of highly polar pesticides in food matrices using hydrophilic interaction liquid chromatography and mass spectrometry. $J$. Chromatogr. A 2019, 1594, 93-104. [CrossRef]

35. Britzi, M.; Schwartsburd, F. Development and validation of a high-throughput method for the determination of eight non-steroidal anti-inflammatory drugs and chloramphenicol in milk, using liquid chromatography-tandem mass spectroscopy. Int. J. Analyt. Bioanalyt. Methods 2019, 1, 005.

36. Shishov, A.; Nechaeva, D.; Bulatov, A. HPLC-MS/MS determination of non-steroidal anti-inflammatory drugs in bovine milk based on simultaneous deep eutectic solvents formation and its solidification. Microchem. J. 2019, 150, 104080. [CrossRef] 
37. Wang, Y.; Ou, Y.; Xie, S.; Chen, D.; Wang, X.; Pan, Y.; Wang, Y.; Huang, L.; Cheng, G.; Qu, W.; et al. Magnetic graphene solid-phase extraction for the determination of 47 kinds of non-steroidal anti-inflammatory drug residues in animal food with liquid chromatography tandem mass spectrometry. Food Anal. Methods 2019, 12, 1346-1368. [CrossRef]

38. Kim, M.K.; Kim, N.S.; Kwon, H.J.; Ha, S.Y.; Kim, H.S.; Kim, J.W. Development of a simultaneous multi-residue analysis for screening and confirmation of 7 veterinary drugs in bovine milk by LC-MSMS. J. Prev. Vet. Med. 2019, 43, 68-73. [CrossRef]

39. Li, M.; Liang, X.; Guo, X.; Di, X.; Jiang, Z. Enantiomeric separation and enantioselective determination of some representive non-steroidal anti-inflammatory drug enantiomers in fish tissues by using chiral liquid chromatography coupled with tandem mass spectrometry. Microchem. J. 2020, 153, 104511. [CrossRef]

40. Liang, S.; Jian, N.; Cao, J.; Zhang, H.; Li, J.; Xu, Q. Rapid, simple and green solid phase extraction based on polyaniline nanofibers-mat for detecting non-steroidal anti-inflammatory drug residues in animal-origin food. Food Chem. 2020, 328, 127097. [CrossRef]

41. Timofeeva, I.; Stepanova, K.; Shishov, A.; Nugbienyo, L.; Moskvin, L.; Bulatov, A. Fluoroquinolones extraction from meat samples based on deep eutectic solvent formation. J. Food Compos. Anal. 2020, 93, 103589. [CrossRef]

42. Alqahtani, S.S.; Humaid, D.M.B.; Alshail, S.H.; AlShammari, D.T.; Al-Showiman, H.; Alzoman, N.Z.; Maher, H.M. Development and validation of a high performance liquid chromatography/diode array detection method for estrogen determination: Application to residual analysis in meat products. Open Chem. 2020, 18, 995-1010. [CrossRef]

43. Caulfield, M.P.; Padula, M.P. HPLC MS-MS analysis shows measurement of corticosterone in egg albumen is not a valid indicator of chicken welfare. Animals 2020, 10, 821. [CrossRef]

44. Han, X.; Liu, D. Detection and analysis of 17 steroid hormones by ultra-high-performance liquid chromatography-electrospray ionization mass spectrometry (UHPLC-MS) in different sex and maturity stages of Antarctic krill (Euphausia superba Dana). PLoS ONE 2019, 14, e0213398. [CrossRef]

45. Yue, C.S.; Hong, W.L.; Tan, S.A.S.W.; Loh, K.E.; Liew, Y.C.; Yap, R.E.; Chong, Z.Y.; Chai, J.C. Identification and validation of synthetic phenolic antioxidants in various foods commonly consumed in Malaysia by HPLC. Indones. J. Chem. 2019, 19, 907-919. [CrossRef]

46. Gbylik-Sikorska, M.; Gajda, A.; Burmańczuk, A.; Grabowski, T.; Posyniak, A. Development of a UHPLC-MS/MS method for the determination of quercetin in milk and its application to a pharmacokinetic study. J. Vet. Res. 2019, 63, 87-91. [CrossRef]

47. Velkoska-Markovska, L.; Jankulovska, M.S.; Petanovska-Ilievska, B.; Hristovski, K. Development and validation of RPLC-UV method for determination of chlorogenic acid in green coffee. Acta Chromatogr. 2020, 32, 34-38. [CrossRef]

48. Atanacković Krstonošić, M.; Cvejić Hogervorst, J.; Mikulić, M.; Gojković-Bukarica, L. Development of HPLC method for determination of phenolic compounds on a core shell column by direct injection of wine samples. Acta Chromatogr. 2020, 32, 134-138. [CrossRef]

49. Kurjogi, M.; Issa Mohammad, Y.H.I.; Alghamdi, S.; Abdelrahman, M.; Satapute, P.; Jogaiah, S. Detection and determination of stability of the antibiotic residues in cow's milk. PLoS ONE 2019, 14, e0223475. [CrossRef]

50. Dinh, Q.T.; Munoz, G.; Duy, S.V.; Do, D.T.; Bayen, S.; Sauvé, S. Analysis of sulfonamides, fluoroquinolones, tetracyclines, triphenylmethane dyes and other veterinary drug residues in cultured and wild seafood sold in Montreal, Canada. J. Food Compos. Anal. 2020, 94, 103630. [CrossRef]

51. Uenoyama, R.; Miyazaki, M.; Miyazaki, T.; Shigeno, Y.; Tokairin, Y.; Konno, H.; Yamashita, T. LC-ESI-MS/MS quantification of carnosine, anserine, and balenine in meat samples. J. Chromatogr. B 2019, 1132, 121826. [CrossRef] [PubMed]

52. Przybylska, A.; Bazylak, G.; Kosicki, R.; Altyn, I.; Twaruzek, M.; Grajewski, J.; Soltys-Lelek, A. Advantageous extraction, cleanup, and UHPLC-MS/MS detection of patulin mycotoxin in dietary supplements and herbal blends containing hawberry from Crataegus spp. J. Anal. Methods Chem. 2019. [CrossRef] [PubMed]

53. Zhao, M.; Shao, H.; Ma, J.; Li, H.; He, Y.; Wang, M.; Jin, M.; Wang, J.; Abd El-Aty, A.M.; Hacımüftüoğlu, A.; et al. Preparation of core-shell magnetic molecularly imprinted polymers for extraction of patulin from juice samples. J. Chromatogr. A 2020, 1615, 460751. [CrossRef] [PubMed]

54. Dural, E. Monitorization of patulin and hydroxymethylfurfural in fruit juices and commercial fruity baby foods by an HPLC-DAD method. Rev. Roum. Chim. 2020, 65, 191-200. [CrossRef]

55. Hassan, N.H.; Othman, H.I.A.A.; Abdul Malek, N.R.; Zulkurnain, M.; Saad, B.; Wong, Y.F. Simultaneous quantitative assessment of ochratoxin A, patulin, 5-Hydroxymethylfurfural, and bisphenol A in fruit drinks using HPLC with Diode Array-Fluorimetric Detection. Foods 2020, 9, 1633. [CrossRef]

56. Hussain, S.; Asi, M.R.; Iqbal, M.; Akhtar, M.; Imran, M.; Ariño, A. Surveillance of patulin in apple, grapes, juices and value-added products for sale in Pakistan. Foods 2020, 9, 1744. [CrossRef]

57. Hussain, S.; Asi, M.R.; Iqbal, M.; Khalid, N.; Wajih-ul-Hassan, S.; Ariño, A. Patulin mycotoxin in mango and orange fruits, juices, pulps, and jams marketed in Pakistan. Toxins 2020, 12, 52. [CrossRef]

58. Lien, K.W.; Ling, M.P.; Pan, M.H. Probabilistic risk assessment of patulin in imported apple juice and apple-Containing beverages in Taiwan. J. Sci. Food Agric. 2020, 100, 4776. [CrossRef]

59. Cheung, Y.; Meenu, M.; Yu, X.; Xu, B. Phenolic acids and flavonoids profiles of commercial honey from different floral sources and geographic sources. Int. J. Food Prop. 2019, 22, 290-308. [CrossRef]

60. Radović, M.; Dragan Milatović, D.; Tešić, Ž.; Tosti, T.; Gašić, U.; Dojčinović, B.; Dabić Zagorac, D. Influence of rootstocks on the chemical composition of the fruits of plum cultivars. J. Food Compos. Anal. 2020, 92, 103480. [CrossRef] 
61. Pepe, G.; Salviati, E.; Rapa, S.F.; Ostacolo, C.; Cascioferro, S.; Manfra, M.; Autore, G.; Marzocco, S.; Campiglia, P. Citrus sinensis and Vitis vinifera protect cardiomyocytes from doxorubicin-induced oxidative stress: Evaluation of onconutraceutical potential of vegetable smoothies. Antioxidants 2020, 9, 378. [CrossRef] [PubMed]

62. Kyraleou, M.; Kallithraka, S.; Gkanidi, E.; Koundouras, S.; Mannion, D.T.; Kilcawley, K.N. Discrimination of five Greek red grape varieties according to the anthocyanin and proanthocyanidin profiles of their skins and seeds. J. Food Compos. Anal. 2020, 92, 103547. [CrossRef]

63. Sochorova, L.; Klejdus, B.; Baro, M.; Jurikova, T.; Mlcek, J.; Sochor, J.; Ercisli, S.; Kupe, M. Assessment of antioxidants by HPLC-MS in grapevine seeds. Acta Sci. Pol. Hortorum Cultus. 2019, 18, 17-28. [CrossRef]

64. El-Nakhel, C.; Pannico, A.; Graziani, G.; Kyriacou, M.C.; Giordano, M.; Ritieni, A.; De Pascale, S.; Rouphael, Y. Variation in macronutrient content, phytochemical constitution and In Vitro antioxidant capacity of green and red butterhead lettuce dictated by different developmental stages of harvest maturity. Antioxidants 2020, 9, 300. [CrossRef]

65. Cirilli, R.; Gallo, F.R.; Multari, G.; Palazzino, G.; Mustazza, C.; Panusa, A. Study of solvent effect on the stability of isothiocyanate iberin, a breakdown product of glucoiberin. J. Food Compos. Anal. 2020, 92, 103515. [CrossRef]

66. Liu, R.; Choi, H.S.; Kim, S.L.; Kim, J.H.; Yun, B.S.; Lee, D.S. 6-Methoxymellein isolated from carrot (Daucus carota L.) targets breast cancer stem cells by regulating NF- $\mathrm{KB}$ signaling. Molecules 2020, 25, 4374. [CrossRef] [PubMed]

67. Vargas-Pérez, M.; Domínguez, I.; Egea González, F.J. Application of full scan gas chromatography high resolution mass spectrometry data quantify targeted-pesticide residues and to screen for additional substances of concern in fresh-food commodities. $J$. Chromatogr. A 2020, 1622, 461118. [CrossRef] [PubMed]

68. Wang, X.; Wang, Z.; Di, S.; Xue, X.; Jin, Y.; Qi, P.; Wang, X.; Han, L.; Xiao, Y.; Min, S. Determination of 14 lipophilic pesticide residues in raw propolis by selective sample preparation and gas chromatography-tandem mass spectrometry. Food Anal. Methods 2020, 13, 1726-1735. [CrossRef]

69. Moinfar, S.; Jamil, L.A.; Sami, H.Z. Determination of organophosphorus pesticides in juice and water by modified continuous sample drop flow microextraction combined with gas chromatography-mass spectrometry. Food Anal. Methods 2020, 13, 1050-1059. [CrossRef]

70. Azzouz, A.; Colón, L.P.; Hejji, L.; Ballesteros, E. Determination of alkylphenols, phenylphenols, bisphenol A, parabens, organophosphorus pesticides and triclosan in different cereal-based foodstuffs by gas chromatography-mass spectrometry. Anal. Bioanal. Chem. 2020, 412, 2621-2631. [CrossRef]

71. Zhao, Y.; Hou, X.; Qin, D.; Liu, D. Dispersive liquid-liquid microextraction method for the simultaneous determination of four isomers of hexachlorocyclohexane and six pyrethroid pesticides in milk by gas chromatography electron capture detector. Food Anal. Methods 2020, 13, 370-381. [CrossRef]

72. Li, S.; Yu, P.; Zhou, C.; Tong, L.; Li, D.; Yu, Z.; Zhao, Y. Analysis of pesticide residues in commercially available chenpi using a modified QuEChERS method and GC-MS/MS determination. J. Pharm. Anal. 2020, 10, 60-69. [CrossRef]

73. Wolecki, D.; Caban, M.; Pazdro, K.; Mulkiewicz, E.; Stepnowski, P.; Kumirska, J. Simultaneous determination of non-steroidal anti-inflammatory drugs and natural estrogens in the mussels. Mytilus Edulis Trossulus. Talanta 2019, 200, 316-323. [CrossRef]

74. Lim, H.H.; Shin, H.S. In-solution derivatization and detection of glyoxal and methylglyoxal in alcoholic beverages and fermented foods by headspace solid-phase microextraction and gas chromatography-mass spectrometry. J. Food Compos. Anal. 2020, 92, 103584. [CrossRef]

75. Lee, S.; Lim, D.K.; Baek, S.Y.; Seo, D.; Park, J.S.; Kwak, B.M.; Won, J.; Lee, J.; Kim, B. Quantitative analyses of essential fatty acids in cereals and green vegetables by isotope dilution-gas chromatography/mass spectrometry. J. Anal. Sci. Technol. 2020, 11, 37. [CrossRef]

76. Iko Afe, O.H.; Anihouvi, D.G.; Assogba, M.F.; Anihouvi, E.L.; Kpoclou, Y.E.; Douny, C.; Mahillon, J.; Anihouvi, V.B.; Scippo, M.L.; Hounhouigan, D.J. Consumption and nutritional quality of grilled pork purchased from open road-side restaurants of Benin. J. Food Compos. Anal. 2020, 92, 103549. [CrossRef]

77. Choi, E.; Kim, B.H. A comparison of the fat, sugar, and sodium contents in ready-to-heat type home meal replacements and restaurant foods in Korea. J. Food Compos. Anal. 2020, 92, 103524. [CrossRef]

78. Jarukas, L.; Kuraite, G.; Baranauskaite, J.; Marksa, M.; Bezruk, I.; Ivanauskas, L. Optimization and validation of the GC/FID method for the quantification of fatty acids in bee products. Appl. Sci. 2021, 11, 83. [CrossRef]

79. Fruehwirth, S.; Egger, S.; Flecker, T.; Ressler, M.; Firat, N.; Pignitter, M. Acetone as indicator of lipid oxidation in stored margarine. Antioxidants 2021, 10, 59. [CrossRef] [PubMed]

80. Shahnawaz, A.; Rattanpal, H.S.; Gul, K.; Rouf Ahmad Dar, R.A.; Sharma, A. Chemical composition, antioxidant activity and GC-MS analysis of juice and peel oil of grapefruit varieties cultivated in India. J. Int. Agric. 2019, 18, 1634-1642.

81. Szabo, K.; Dulf, F.V.; Teleky, B.-E.; Eleni, P.; Boukouvalas, C.; Krokida, M.; Kapsalis, N.; Rusu, A.V.; Socol, C.T.; Vodnar, D.C. Evaluation of the bioactive compounds found in tomato seed oil and tomato peels influenced by industrial heat treatments. Foods 2021, 10, 110. [CrossRef] [PubMed]

82. Migas, P.; Stempka, N.; Krauze-Baranowska, M. The use of thin-layer chromatography in the assessment of the quality of lutein-containing dietary supplements. J. Planar Chromatogr. Mod. TLC 2020, 33, 11-18. [CrossRef]

83. Yogeswara, I.B.A.; Kittibunchakul, S.; Rahayu, E.S.; Domig, K.J.; Haltrich, D.; Nguyen, T.H. Microbial production and enzymatic biosynthesis of $\gamma$-aminobutyric acid (GABA) using Lactobacillus plantarum FNCC 260 isolated from indonesian fermented foods. Processes 2021, 9, 22. [CrossRef] 
84. Mitema, A.; Feto, N.A.; Rafudeen, M.S. Development and validation of TOF/Q-TOF MS/MS, HPLC method and in vitro bio-strategy for aflatoxin mitigation. Food Addit. Contam. Part A 2020. [CrossRef] [PubMed]

85. Kang, Y.; Wu, T.; Chen, W.; Li, L.; Du, Y. A novel metastable state nanoparticle-enhanced Raman spectroscopy coupled with thin layer chromatography for determination of multiple pesticides. Food Chem. 2019, 270, 494-501. [CrossRef]

86. Gaweł, M.; Kiljanek, T.; Niewiadomska, A.; Semeniuk, S.; Goliszek, M.; Burek, O.; Posyniak, A. Determination of neonicotinoids and 199 other pesticide residues in honey by liquid and gas chromatography coupled with tandem mass spectrometry. Food Chem. 2019, 282, 36-47. [CrossRef] [PubMed] 